ESAIM: PROCEEDINGS AND SURVEYS, December 2015, Vol. 52, p. 25-46

S. Dellacherie, F. Dubois, S. Fauve, R. Gatignol, Editors

\title{
TAYLOR EXPANSION METHOD FOR ANALYZING BOUNCE-BACK BOUNDARY CONDITIONS FOR LATTICE BOLTZMANN METHOD
}

\author{
François Dubois ${ }^{1,2}$, Pierre Lallemand ${ }^{3}$ And Mohamed Mahdi Tekitek ${ }^{4,5}$
}

\begin{abstract}
In this contribution, we propose a new method of analysis for the lattice Boltzmann scheme's boundary conditions. We develop this approach for bounce-back with a given velocity between two mesh points. We develop a "accordion" test case for linear stationary Stokes problem with an analytic expression. Numerical experiment show a good agreement at first and second order accuracy. This study has been also applied to the computation of momentum transfer.

Résumé. Dans cette contribution, nous proposons une nouvelle méthode d'analyse des conditions aux limites pour les schémas de Boltzmann sur réseau. Nous développons cette approche pour une condition de rebond avec une donnée de vitesse imposée. Nous développons un cas test "accordéon" pour le problème de Stokes stationnaire et en explicitons la solution analytique. Nos expériences numériques montrent un bon accord entre solution approchée numérique et solution analytique, à l'ordre un et à l'ordre deux. L'étude précédente est également appliquée pour le calcul du transfert d'impulsion à la paroi.
\end{abstract}

\section{INTRODUCTION}

In this contribution, we study boundary conditions for lattice Boltzmann schemes. We refer to Ginzburg and Adler [9], Zou and He [21], Bouzidi et al. [1], d'Humières and Ginzburg [13] for bounce-back boundary condition, anti-bounce-back boundary condition and the determination of so-called "quartic" parameters to enforce the precision of the scheme. The boundary is also an essential location for momentum exchange. In fact 1994 Ladd [16] the Momentum Exchange Algorithm (MEA) which models the fluid-boundary interaction based on simple particle dynamics. This method has been used in numerical simulations [19,20]. He et al. [10], focus on the lattice Boltzmann for a general curvilinear coordinate and they use interpolation to compute different moments transfer. Inamuro et al. [14], proposed another method to models the fluid-solid interaction using asymptotic analysis. More recently, [2] investigated in details the theoretical proprieties of the MEA and [3] proposed a corrected MEA to ameliorate the method in the case of moving particles. It is also possible to work with specific finite difference stencil for the momentum flux at the wall, using the equivalent finite difference stencil approach of Junk and Klar [15].

In a previous contribution [8], we have used the Taylor expansion method to analyze precisely the numerical behaviour of a lattice Boltzmann scheme with an external force, focusing on a precise determination of the

\footnotetext{
1 Structural Mechanics and Coupled Systems Laboratory, CNAM Paris, France.

2 Department of Mathematics, University Paris-Sud, Orsay, France.

3 Beijing Computational Science Research Center, Beijing, China.

4 Faculty of Sciences of Tunis, University of Tunis El Manar, Tunis, Tunisia.

5 Department of Mathematics, Conservatoire National des Arts et Métiers, 292, rue Saint Martin, Paris, 75003, France.
}

(C) EDP Sciences, SMAI 2015 
numerical boundary. In this contribution, we follow the same idea to recover precise physical data from linear combinations of the particle distributions and appropriate numerical corrections.

To fix the ideas, we consider an homogeneous bounce-back boundary condition for a fluid flow simulated with a D2Q9 scheme and a bounce-back boundary condition to enforce zero velocity at the boundary. The question is to capture with a good approximation the wall friction $\tau \equiv \mu \frac{\partial u}{\partial y}$ for a boundary located along an horizontal boundary $y=0$. When we analyze the transfer of momentum due to particles crossing the boundary, a natural expression is given by $T_{H} \equiv f_{5}-f_{6}+f_{7}-f_{8}$. Then we expand the previous expression as powers of the mesh size. In previous works [7] and [8] we used the expansion of the internal scheme to analyze the bounce-back. In this contribution, we consider the algebraic details of the numerical scheme for a boundary node.

In this contribution, we first propose an analysis of bounce-back boundary condition (Section 1). Then we validate it for two test cases: the Poiseuille test case and the accordion test case. The second test case is not usual and is presented in detail. In Section 3, we propose a development for parallel moment transfer using directly the Lattice Boltzmann variables without adding any interpolation. This method is the same idea to MEA [17] which is for interaction between fluid and boundary. Finally, we give a Taylor expansion of our method and a first numerical validation.

\section{AnAlysis of BOUnCE-BACK BOUndARY CONDITIONS}

\section{- Lattice Boltzmann schemes}

A lattice Boltzmann scheme is defined through the evolution of a population $\left\{f_{i}\right\}$ of $q$ discrete velocities where time and space are discretized. The population evolves in a succession of collision and propagation steps on the nodes of a regular lattice in $d$ dimensions, parametrized by a spatial step $\Delta x$. The time step $\Delta t$ is determined thanks to the acoustic scale $\lambda\left(i . e . \Delta t=\frac{\Delta x}{\lambda}\right)$. For the $\mathrm{D} d \mathrm{Q} q$ scheme, we note $\left(v_{j}\right)_{0 \leq j \leq q-1}$ the set of $q$ velocities and we assume that for each node $x$ and each velocity $v_{j}$, the vertex $x-v_{j} \Delta t$ is also a node of the lattice. The components of the velocity $v_{j}$ are denoted by $v_{j}^{\alpha}$. So a time step of a lattice Boltzmann scheme can be written as:

$$
f_{i}(x, t+\Delta t)=f_{i}^{*}\left(x-v_{i} \Delta t, t\right), \quad 0 \leq i \leq q-1,
$$

where the superscript $*$ denotes post-collision quantities. The above equation is composed by two fundamental steps : advection and collision.

- The advection step corresponds to a method of characteristics with the Courant-Friedrichs-Levy (CFL) number equal to 1 for the advection with velocity $v_{j}$. The particles move from a lattice node $x$ to either itself (for $v_{0}=0$ ) or one of the $q-1$ neighbors $x_{j}=x+v_{j} \Delta t$ for velocities $v_{j} \neq 0$. For the D2Q9 the velocities are presented in the Figure 1.

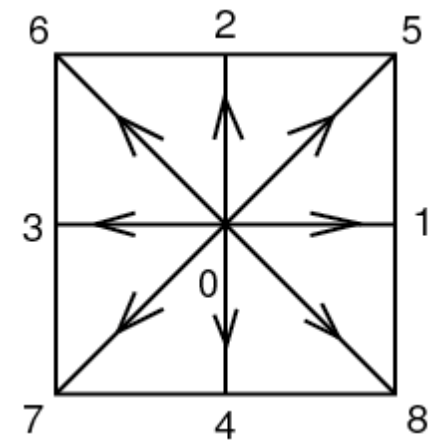

FIGURE 1. Stencil for the D2Q9 lattice Boltzmann scheme.

As proposed by d'Humières [12], we introduce the moments $m$ defined by $m=M f$ where $M$ is a given invertible matrix. For the D2Q9 lattice Boltzmann scheme, the matrix $M$ is made explicit by relation (28) in 
the Appendix. So in the moment space it is easier to describe the collision step. The moment vector is composed of two kinds of quantities: the first one of conserved variables $W \in \mathbb{R}^{N}$ which are not affected by the collision step when there is no forcing term. The second one of non conserved quantities $Y$ relax in the collision step. So the moment vector becomes $m=(W, Y)^{\mathrm{t}}$.

- The relaxation step is performed in the moment space as follows:

$$
Y^{*}=(\operatorname{Id}-S) Y+S Y^{\mathrm{eq}},
$$

where Id is the identity matrix and $S$ is the diagonal matrix of the relaxation rates $s_{k}, N+1 \leq k \leq q-1$ with $0<s_{k}<2$. The equilibrium distribution is given in the linear case by the relation $Y^{\text {eq }}=E W$, where $E$ is a fixed matrix with $q-N$ lines and $N$ columns. Thus the relaxation is simply described by :

$$
m_{k}^{*}=\left(1-s_{k}\right) m_{k}+s_{k} m_{k}^{e q}, \quad N+1 \leq k \leq q-1
$$

For the D2Q9 scheme, the equilibrium values of the momenta and matrix of the relaxation $S$ are made explicit respectively by the relations (29) and (30) in the Appendix. They introduce two coefficients denoted by $a_{2}$ and $a_{3}$.

Using Taylor expansion (see [5]), the D2Q9 Boltzmann scheme defined by (1), (28), (29) and (30), admits the following equivalent partial differential equations :

$$
\left\{\begin{array}{l}
\partial_{t} \rho+\partial_{x} j_{x}+\partial_{y} j_{y} \\
\partial_{t} j_{x}+\lambda^{2}\left(\frac{4+a_{2}}{6}\right) \partial_{x} \rho-\Delta t \frac{\lambda^{2}}{6}\left[\left(\frac{1}{s_{3}}-\frac{1}{2}\right) \partial_{x}\left(\partial_{x} j_{x}+\partial_{y} j_{y}\right)+\left(\frac{1}{s_{4}}-\frac{1}{2}\right) \Delta j_{x}\right]=\begin{array}{l}
\mathrm{O}\left(\Delta t^{2}\right) \\
\partial_{t} j_{y}+\lambda^{2}\left(\frac{4+a_{2}}{6}\right) \partial_{y} \rho-\Delta t \frac{\lambda^{2}}{6}\left[\left(\frac{1}{s_{3}}-\frac{1}{2}\right) \partial_{y}\left(\partial_{x} j_{x}+\partial_{y} j_{y}\right)+\left(\frac{1}{s_{4}}-\frac{1}{2}\right) \Delta j_{y}\right]=
\end{array}=\mathrm{O}\left(\Delta t^{2}\right)
\end{array}\right.
$$

where $\Delta j_{x}$ and $\Delta j_{y}$ are the Laplace operator of $j_{x}$ and $j_{y}$ respectively. In the case of the steady state (i.e. $\left.\partial_{t} \equiv 0\right)$, the above equations becomes

$$
\partial_{x} j_{x}+\partial_{y} j_{y}=0, \quad \partial_{x} p-\nu \Delta j_{x}=0, \quad \partial_{y} p-\nu \Delta j_{y}=0
$$

up to second order accuracy [4]. This system is exactly the system of Stokes equations with $\nu=\frac{\lambda^{2} \Delta t}{6}\left(\frac{1}{s_{4}}-\frac{1}{2}\right)$. Moreover, we have the relation

$$
p=\lambda^{2} \frac{4+a_{2}}{6} \rho
$$

to take into account the compressible effects of the initial model.

\section{- Boundary conditions}

Let us consider, to fix the ideas, the bottom boundary as described in Figure 2. In this case the distributions $f_{i}^{*}\left(x-v_{i} \Delta t\right)$ for $i \in\{2,5,6\} \equiv \mathcal{B}$ are unknown. So we can not apply the scheme (1) for $i \in \mathcal{B}$ but the scheme is still well defined for others values of $i$ (i.e. $i \in\{0,1,3,4,7,8\}$ ). The bounce-back boundary condition [1] is based on the fact that for any internal vertex $x$, we have the following relations:

$$
\left\{\begin{array}{l}
f_{2}^{*}(x)-f_{4}^{*}(x)=\frac{2}{3 \lambda} j_{y}(x)+\mathrm{O}(\Delta t) \\
f_{5}^{*}(x)-f_{7}^{*}(x)=\frac{1}{6 \lambda}\left(j_{x}+j_{y}\right)(x)+\mathrm{O}(\Delta t) \\
f_{6}^{*}(x)-f_{8}^{*}(x)=\frac{1}{6 \lambda}\left(-j_{x}+j_{y}\right)(x)+\mathrm{O}(\Delta t)
\end{array}\right.
$$

The above equations are obtained if we suppose that we have equilibrium at the internal node $x$.

Let $\left(J_{x}, J_{y}\right)(x, t)$ be a given function on the boundary which is a non-homogeneous velocity to impose. We note 


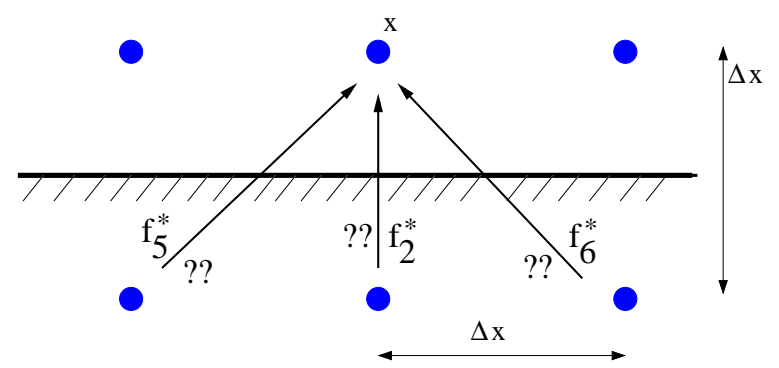

FIGURE 2. The question of the boundary scheme for the D2Q9 lattice Boltzmann scheme on a boundary located at $y=0$.

here that $J_{x}$ (respectively $J_{y}$ ) is not identical to the velocity moments $j_{x}$ and $j_{y}$ defined by the relations

$$
j_{x}=\sum_{j} v_{j}^{x} f_{j}, \quad j_{y}=\sum_{j} v_{j}^{y} f_{j}
$$

and numbered with the indexes 1 and 2 for the D2Q9 scheme, as given in the relation (28). In order to impose a given field $\left(J_{x}, J_{y}\right)$ on the boundary (see e.g. [6]), the bounce-back condition is defined as:

$$
\left\{\begin{array}{l}
f_{2}(x, t+\Delta t)=f_{4}^{*}(x)+\frac{2}{3 \lambda} J_{y}\left(x, t+\frac{\Delta t}{2}\right), \\
f_{5}(x, t+\Delta t)=f_{7}^{*}(x)+\frac{1}{6 \lambda}\left(J_{x}+J_{y}\right)\left(x-\frac{\Delta x}{2}, t+\frac{\Delta t}{2}\right), \\
f_{6}(x, t+\Delta t)=f_{8}^{*}(x)+\frac{1}{6 \lambda}\left(-J_{x}+J_{y}\right)\left(x+\frac{\Delta x}{2}, t+\frac{\Delta t}{2}\right) .
\end{array}\right.
$$

The bounce-back numerical boundary condition is exact up to order one in space; we refer to Ginzburg and d'Humières [13] for more precise boundary conditions.

\section{- An analysis with the Taylor expansion method}

In a previous work [8] we have used the Taylor expansion method to analyze precisely the numerical behavior of the lattice Boltzmann scheme. The aim here is to use the same idea to analyze precisely the boundary condition. Rewriting the bounce-back in general form:

$$
f_{j}^{*}(x, t+\Delta t)=f_{\ell}^{*}(x, t)+\xi_{j}\left(x^{\prime}, t^{\prime}\right), \quad j \in \mathcal{B},
$$

where $\ell$ is opposite of $j$ in the sense of $v_{j}+v_{\ell}=0$, and $\xi_{j}\left(x^{\prime}, t^{\prime}\right)$ is the given source term associated to the velocity on the boundary. By introducing a table $T_{j, \ell}$, the lattice Boltzmann scheme for a boundary node $x$ is given by

$$
f_{j}(x, t+\Delta t)=\sum_{\ell} T_{j, \ell} f_{\ell}^{*}(x, t)+\xi_{j}+\sum_{\ell} U_{j, \ell} f_{\ell}^{*}\left(x-v_{j} \Delta t, t\right),
$$

where the matrix $U_{j, \ell}=1$ if $\ell=j \notin \mathcal{B}$ and $U_{j, \ell}=0$ if not. The matrix $T$ describes the "internal" numerical scheme (7) whereas the matrix $U$ allows us to take into account the bounce-back boundary scheme (6). For the particular bottom boundary with the D2Q9 scheme as presented in Figure 2, the table $T$ is made explicit by the relation (32) in the Appendix. If the index $j$ does not belong in the boundary set $\mathcal{B}$, i.e. $j \notin \mathcal{B}$, the above equation (8) becomes $f_{j}(x, t+\Delta t)=f_{i}^{*}\left(x-v_{j} \Delta t, t\right)$ and we recover the internal scheme presented at the relation (1). Then we move to the space of moments $m: m_{k}=\sum_{j} M_{k j} f_{j}$. For a linear relaxation, the collision step $m \longrightarrow m^{*}$ is given by: $m_{\ell}^{*}=\sum_{k}\left(J_{0}\right)_{\ell, k} m_{k}$ where the matrix $J_{0}$ is given in Lallemand and Luo [18] (see also our contribution [5] and the relation (31) of the Appendix in the D2Q9 scheme developed in this contribution). 
With an implicit summation on the repeated indices, the moment vector the lattice Boltzmann scheme on the boundary node $x$ can be evaluated as follows :

$$
m_{k}(x, t+\Delta t)=\left(M T M^{-1}\right)_{k, \ell} m_{\ell}^{*}(x, t)+M_{k, \ell} U_{\ell, j} M_{j, p}^{-1} m_{p}^{*}\left(x-v_{j} \Delta t, t\right)+M_{k, j} \xi_{j} .
$$

We introduce the linearized collision matrix $J_{0}$ and we get:

$$
m_{k}(x, t+\Delta t)=\left(M T M^{-1} J_{0}\right)_{k, \ell} m_{\ell}(x, t)+\left(M_{k, \ell} U_{\ell, j} M_{j, p}^{-1}\left(J_{0}\right)_{p, q}\right) m_{q}\left(x-v_{j} \Delta t, t\right)+M_{k, j} \xi_{j} .
$$

We perform a Taylor expansion of the above relation (9) up to order 0,1 and 2 to analyze the truncation error of the lattice Boltzmann scheme with a boundary scheme, i.e. the bounce-back condition in the present study.

\section{- Proposition 1. Taylor expansion at order 0.}

We introduce the matrix

$$
K \equiv \operatorname{Id}-M(T+U) M^{-1} J_{0} .
$$

For a bounce-back boundary scheme (9), the moments $m^{0}$ at order zero relatively to the discretization step $\Delta t$ satisfy the following relation:

$$
K m^{0}=M \xi \text {. }
$$

\section{Proof of Proposition 1.}

Just expand the relation (9) at order zero. Then the relation (11) is clear and it is also the case for the algebraic form (10) of the matrix $K$.

- For the D2Q9 scheme, the matrix $K$ is singular and the dimension of its kernel is equal to 1 . We have one compatibility relation to satisfy which is a linear combination of the equivalent equations of the internal scheme, as given by the relation (34) for the bounce-back condition for the D2Q9 scheme. Then the solution of (11) at order zero can be given as

$$
m^{0}=\left(\rho, J_{x}, J_{y}, a_{2} \lambda^{2} \rho, 0,0,-\lambda^{2} J_{x},-\lambda^{2} J_{y}, \lambda^{4} a_{3} \rho\right)^{\mathrm{t}},
$$

where $\rho$ is a free parameter that has to be fixed by the context. In particular the density can be considered expanded as $\rho=\rho^{0}+\Delta t \rho^{1}+\ldots$. We do not give this expansion in the following and interpret the parameter $\rho$ as the density at the node point. We refer also to the relation (37) in the Appendix. This relation describes formally the equilibrium state relative to the density $\rho$ and the given momentum $\left(J_{x}, J_{y}\right)$.

\section{- Proposition 2. Taylor expansion at order 1.}

If we state that the datum $\xi$ introduced in (7) can be expanded up to second order accuracy, i.e. $\xi \equiv \xi^{0}+\Delta t \xi^{1}+\frac{1}{2} \Delta t^{2} \xi^{2}$, we introduce the two matrices $B^{1}$ and $B^{2}$ according to

$$
B_{k, p}^{\alpha}=\sum_{\ell, j, q} M_{k, \ell} U_{\ell, j} v_{j}^{\alpha} M_{j, q}^{-1}\left(J_{0}\right)_{q, p}, \quad 1 \leq k, p \leq q-1,
$$

for $\alpha=1,2$. Then the moments $m \equiv m^{0}+\Delta t m^{1}+\mathrm{O}\left(\Delta t^{2}\right)$ for the bounce-back scheme up to order one are solution of the equation

$$
K m=M \xi^{0}+\Delta t\left[M \xi^{1}-\partial_{t} m-B^{\alpha} \partial_{\alpha} m\right]+\mathrm{O}\left(\Delta t^{2}\right) .
$$

The moment $m^{0}$ is given thanks to the previous proposition and the component $m^{1}$ is formally given by the relation

$$
K m^{1}=\left(M \xi^{1}-\partial_{t} m^{0}-B^{\alpha} \partial_{\alpha} m^{0}\right) .
$$

\section{Proof of Proposition 2.}

We expand the relation (9) up to first order: $m_{k}(x, t)+\Delta t \partial_{t} m_{k}+\mathrm{O}\left(\Delta t^{2}\right)=\left(M T M^{-1} J_{0}\right)_{k, \ell} m_{\ell}(x, t)+$ $\left(M_{k, \ell} U_{\ell, j} M_{j, p}^{-1}\left(J_{0}\right)_{p, q}\right)\left(m_{q}(x, t)-v_{j}^{\alpha} \Delta t \partial_{\alpha} m_{q}(x, t)\right)+M_{k, j}\left(\xi_{j}+\Delta t \xi_{j}^{1}\right)+\mathrm{O}\left(\Delta t^{2}\right)$. With the definition (10) of the matrix $K$ and the introduction (12) of the matrices $B^{\alpha}$ the relation (13) is clear. When we introduce the 
formal expansion $m=m^{0}+\Delta t m^{1}+\mathrm{O}\left(\Delta t^{2}\right)$ inside the relation (13), the expression (14) of the first order term $m^{1}$ is obtained without difficulty.

\section{- Proposition 3. Taylor expansion at order 2.}

We consider the second order matrices $\widetilde{B}^{\alpha, \beta}$ defined thanks to

$$
\widetilde{B}_{k, p}^{\alpha, \beta}=\sum_{k, j, q} M_{k, p} U_{p, j} v_{j}^{\alpha} v_{j}^{\beta} M_{j, q}^{-1}\left(J_{0}\right)_{q, \ell}, \quad 1 \leq \alpha, \beta \leq 2, \quad 0 \leq k, p \leq q-1 .
$$

Then the equivalent equations for bounce-back scheme (9) can be expanded up to order two

$$
K m=M \xi^{0}+\Delta t\left(M \xi^{1}-\partial_{t} m-B^{\alpha} \partial_{\alpha} m\right)+\frac{1}{2} \Delta t^{2}\left(M \xi^{2}-\partial_{t}^{2} m+\widetilde{B}^{\alpha, \beta} \partial_{\alpha} \partial_{\beta} m\right)+\mathrm{O}\left(\Delta t^{3}\right) .
$$

The solution of (16) can be expanded as $m \equiv m^{0}+\Delta t m^{1}+\Delta t^{2} m^{2}+\mathrm{O}\left(\Delta t^{3}\right)$, where $m^{0}$ is solution of (11), $m^{1}$ is solution of (13) and $m^{2}$ is solution of the equation :

$$
K m^{2}=-\partial_{t} m^{1}-B^{\alpha} \partial_{\alpha} m^{1}+\frac{1}{2}\left(M \xi^{2}-\partial_{t}^{2} m^{0}+\widetilde{B}^{\alpha, \beta} \partial_{\alpha} \partial_{\beta} m^{0}\right) .
$$

\section{Proof of Proposition 3.}

The proof is analogous to the proof of Proposition 2. Expand the various terms of the relation (9) up to second order:

$$
\begin{aligned}
m_{k}(x, t) & +\Delta t \partial_{t} m_{k}+\frac{1}{2} \Delta t^{2} \partial_{t}^{2} m_{k}+\mathrm{O}\left(\Delta t^{3}\right)=\left(M T M^{-1} J_{0}\right)_{k, \ell} m_{\ell}(x, t) \\
& +\left(M_{k, \ell} U_{\ell, j} M_{j, p}^{-1}\left(J_{0}\right)_{p, q}\right)\left(m_{q}(x, t)-v_{j}^{\alpha} \Delta t \partial_{\alpha} m_{q}(x, t)+\frac{1}{2} v_{j}^{\alpha} v_{j}^{\beta} \Delta t^{2} \partial_{\alpha \beta} m_{q}(x, t)\right) \\
& +M_{k, j}\left(\xi_{j}^{0}+\Delta t \xi_{j}^{1}+\frac{1}{2} \Delta t^{2} \xi_{j}^{2}\right)+\mathrm{O}\left(\Delta t^{3}\right)
\end{aligned}
$$

and the relation (16) follows. When we insert the expansion $m \equiv m^{0}+\Delta t m^{1}+\Delta t^{2} m^{2}+\mathrm{O}\left(\Delta t^{3}\right)$ inside (16), we obtain at second order in time the relation (17). The proof is completed.

\section{- Proposition 4. Velocity up to order two on boundary node.}

We introduce numbers $\sigma_{k} \equiv\left(\frac{1}{s_{k}}-\frac{1}{2}\right), \quad N+1 \leq k \leq q-1$. For the D2Q9 scheme with the bounce-back boundary conditions described in Figure 2 and relations (6), we have the following expansion for $x$ velocity momentum :

$$
\left\{\begin{array}{c}
j_{x}=J_{x}+\frac{\Delta x}{2} \partial_{y} J_{x}-\Delta t\left(\frac{3}{2}+2 \sigma_{7}\right)\left(\partial_{t} J_{x}+c_{0}^{2} \partial_{x} \rho\right)+\frac{\Delta x}{6} \sigma_{7}\left(3 a_{2}+2 a_{3}+4\right) \partial_{x} \rho \\
+\Delta t^{2}\left[\alpha_{t t} \partial_{t}^{2} J_{x}+\alpha_{t x} \partial_{t} \partial_{x} J_{x}+\alpha_{x x} \partial_{x}^{2} J_{x}+\alpha_{y y} \partial_{y}^{2} J_{x}+\beta_{x t} \partial_{t} \partial_{x} J_{y}\right. \\
\left.+\beta_{x y} \partial_{x} \partial_{y} J_{y}+\gamma_{x t} \partial_{t} \partial_{x} \rho+\gamma_{x y} \partial_{x} \partial_{y} \rho\right]+\mathrm{O}\left(\Delta t^{3}\right)
\end{array}\right.
$$


where

$$
\left\{\begin{array}{c}
\alpha_{t t}=\frac{13}{8}+6 \sigma_{7}\left(1+\sigma_{7}\right), \quad \alpha_{t x}=-\frac{\lambda}{2}\left(3+\sigma_{4}+2 \sigma_{7}\right) \\
\alpha_{x x}=\frac{\lambda^{2}}{24}\left(24 \sigma_{4} \sigma_{7}+8 \sigma_{7} \sigma_{8}+12 \sigma_{4}+15\right), \quad \alpha_{y y}=\frac{\lambda^{2}}{4}\left(1+2 \sigma_{4}\right) \\
\beta_{t x}=\frac{\lambda}{12}\left(12 \sigma_{4} \sigma_{7}-4 \sigma_{7} \sigma_{8}+6 \sigma_{4}-4 \sigma_{7}-9\right), \quad \beta_{x y}=-\frac{\lambda^{2}}{12}\left(12 \sigma_{4} \sigma_{7}-4 \sigma_{7} \sigma_{8}-4 \sigma_{7}-9\right) \\
\gamma_{x t}=-\frac{\lambda^{2}}{12}\left(6 a_{2} \sigma_{7}^{2}+12 a_{3} \sigma_{7}^{2}-24 \sigma_{7}^{2}+4 a_{3} \sigma_{7} \sigma_{8}+2 a_{2} \sigma_{3} \sigma_{7}\right. \\
\left.-3 a_{2} \sigma_{3}+6 a_{3} \sigma_{7}-3 a_{2} \sigma_{7}-40 \sigma_{7}-a_{3}-22\right) \\
\gamma_{x y}=\frac{\lambda^{3}}{36\left(2 \sigma_{7}+1\right)}\left(6 a_{2} \sigma_{3} \sigma_{7}^{2}+4 a_{3} \sigma_{3} \sigma_{7}^{2}+8 \sigma_{3} \sigma_{7}^{2}+24 \sigma_{4} \sigma_{7}^{2}+8 a_{2} \sigma_{7}^{2} \sigma_{8}+8 a_{3} \sigma_{7}^{2} \sigma_{8}\right. \\
-12 a_{3} \sigma_{4} \sigma_{7}^{2}-6 a_{2} \sigma_{4} \sigma_{7}^{2}-16 \sigma_{7}^{2}-4 a_{2} \sigma_{7}^{2}-8 \sigma_{7} \sigma_{8}-12 \sigma_{3} \sigma_{7} \\
-36 \sigma_{4} \sigma_{7}-6 a_{3} \sigma_{3} \sigma_{7}-2 a_{2} \sigma_{7} \sigma_{8}-9 a_{2} \sigma_{3} \sigma_{7}-6 a_{3} \sigma_{7} \sigma_{4}-15 a_{2} \sigma_{4} \sigma_{7} \\
\left.-29 a_{2} \sigma_{7}-6 a_{2} \sigma_{4}-24 \sigma_{4}-92 \sigma_{7}-6 a_{3} \sigma_{7}-9 a_{2}-36\right)
\end{array}\right.
$$

For $y$ velocity momentum we get the following expansion:

$$
\left\{\begin{array}{c}
j_{y}=J_{y}+\frac{\Delta x}{2} \partial_{y} J_{y}-\frac{\Delta t}{2}\left(\partial_{t} J_{y}+c_{0}^{2} \partial_{y} \rho\right) \\
+\Delta t^{2}\left[\delta_{t x} \partial_{t} \partial_{x} J_{x}+\delta_{x y} \partial_{x} \partial_{y} J_{x}+\eta_{t t} \partial_{t}^{2} J_{y}+\eta_{t y} \partial_{t} \partial_{y} J_{y}+\eta_{x x} \partial_{x}^{2} J_{y}+\eta_{y y} \partial_{y}^{2} J_{y}\right. \\
\left.+\zeta_{t y} \partial_{t} \partial_{y} \rho+\zeta_{x x} \partial_{x}^{2} \rho+\zeta_{y y} \partial_{y}^{2} \rho\right]+\mathrm{O}\left(\Delta t^{3}\right)
\end{array}\right.
$$

where

$$
\left\{\begin{array}{c}
\delta_{t x}=-\frac{\lambda}{4}\left(1+2 \sigma_{4}\right), \quad \delta_{x y}=\frac{\lambda^{2}}{4}, \quad \eta_{t t}=\frac{1}{8}, \quad \eta_{t y}=-\frac{\lambda}{6}\left(4+\sigma_{4}\right), \\
\eta_{x x}=\frac{\lambda^{2}}{24}\left(1+4 \sigma_{4}\right), \quad \eta_{y y}=\frac{\lambda^{2}}{12}\left(5+2 \sigma_{4}\right), \quad \zeta_{t y}=\frac{\lambda^{2}}{24}\left(2 a_{2} \sigma_{3}+a_{2}+8\right), \\
\zeta_{x x}=-\frac{\lambda^{3}}{24}\left(1+2 \sigma_{4}\right)\left(a_{2}+4\right), \\
\zeta_{x y}=-\frac{\lambda^{3}}{72\left(1+2 \sigma_{7}\right)}\left(8 \sigma_{3} \sigma_{7}+8 \sigma_{4} \sigma_{7}-2 a_{2} \sigma_{4} \sigma_{7}+4 a_{3} \sigma_{3} \sigma_{7}+6 a_{2} \sigma_{3} \sigma_{7}-4 a_{3} \sigma_{4} \sigma_{7}\right. \\
\left.+2 a_{2} \sigma_{4}+10 a_{2} \sigma_{7}+40 \sigma_{7}+8 \sigma_{4}+5 a_{2}+20\right) .
\end{array}\right.
$$

- The proof of Proposition 4 is easily obtained by formal computation described in the Appendix.

\section{EXPERIMENTAL VALIDATION OF BOUNCE-BACK BOUNDARY EXPANSION}

We consider here, commun MRT D2Q9 [18] linear lattice Boltzmann scheme with the following equilibrium distribution:

$$
m_{3}^{\mathrm{eq}}=a_{2} \rho, \quad m_{4}^{\mathrm{eq}}=m_{5}^{\mathrm{eq}}=0, \quad m_{6}^{\mathrm{eq}}=-\frac{j_{x}}{\lambda}, \quad m_{7}^{\mathrm{eq}}=-\frac{j_{y}}{\lambda}, \quad m_{8}^{\mathrm{eq}}=a_{3} \rho
$$

We take constant value for relaxation rates leading to a constant kinematic shear viscosity $\nu \equiv \frac{\mu}{\rho}$.

\section{- Poiseuille flow}

First case with volumic force: We consider here the flow driven by spatially uniform body force $f_{x}$ instead of a pressure gradient (see [8] for more details). We perform LBM in $\Omega=\left[1, N_{x}\right] \times\left[1, N_{y}\right]$. For boundary 
condition we take periodic boundary condition on the inlet $(i=1)$ and outlet $\left(i=N_{x}\right)$ of the domain. We apply bounce-back on the bottom $(j=1)$ and the top $\left(j=N_{y}\right)$ of the domain to impose homogeneous Dirichlet boundary condition on the velocity $\left(j_{x}=0\right)$. We can have the solid wall $\left(j_{x}=0\right)$ exactly at the position $\frac{\Delta x}{2}$ for

$$
\sigma_{4} \sigma_{7} \equiv\left(\frac{1}{s_{4}}-\frac{1}{2}\right)\left(\frac{1}{s_{7}}-\frac{1}{2}\right)=\frac{3}{16}
$$

as proved in d'Humières et al. [13] and recovered in our contribution [7]. The proof consists just in applying the expansion (18) that can be written in this particular case of a Poiseuille flow:

$$
j_{x}=J_{x}+\frac{\Delta t}{2} \lambda \partial_{y} J_{x}+\Delta t^{2} \lambda^{2} \widetilde{\alpha}_{y y} \partial_{y}^{2} J_{x}
$$

where $\widetilde{\alpha}_{y y}=\frac{1}{48}\left(12-32 \sigma_{4} \sigma_{7}\right)$ is obtained by the equation (18) after replacing the first spacial derivative of $\rho$ by second order derivative of $j_{y}$ obtained with the equivalent equation (3) and the relation (4) between $\rho$ and $p$.

Remark. The solid wall position is exactly $\frac{\Delta x}{2}$ because Poiseuille LBM solution is polynomial of degree two (parabola), as first observed in [11]. Thus all terms in Taylor expansion for higher order than $\left(\Delta t^{2}\right)$ are null.

Second case with pressure boundary condition: Here we apply a so-called "anti-bounce-back" boundary condition at inlet and outlet of the channel to impose pressure $\delta p$ and $-\delta p$ and bounce-back on the bottom $j=1$ and the top $j=N_{y}$ of the domain to impose $j_{x}=0$. The solid wall is exactly at $\frac{\Delta x}{2}$ for the following condition:

$$
\sigma_{4} \sigma_{7}=-\frac{3}{8} \frac{a_{2}+4}{a_{2}+2 a_{3}-4}
$$

as proposed in our previous contribution [7].

Proof: In the case of the Poiseuille flow obtained by a pressure boundary condition (i.e. without using a volumic force) equation (18) becomes:

$$
j_{x}=J_{x}+\frac{\Delta t}{2} \lambda \partial_{y} J_{x}+\frac{\Delta t}{6} \lambda \sigma_{7}\left(3 a_{2}+2 a_{3}+4\right) \partial_{x} \rho+\Delta t^{2} \lambda^{2} \widetilde{\alpha}_{y y} \partial_{y}^{2} J_{x}
$$

where $\widetilde{\alpha}_{y y}=\frac{1}{48}\left(12-32 \sigma_{4} \sigma_{7}\right)$. Because in this case the LBM solution is also polynomial of degree two (parabola). Now by using equivalent equation to replace the expression of $\partial_{x} \rho$ we get:

$$
j_{x}=J_{x}+\frac{\Delta t}{2} \lambda \partial_{y} J_{x}+\Delta t^{2} \lambda^{2}\left[\sigma_{4} \sigma_{7} \frac{3 a_{2}+2 a_{3}+4}{3}+\widetilde{\alpha}_{y y}\right] \partial_{y}^{2} J_{x}
$$

Finally we obtain the proof by taking the coefficient of the term $\partial_{y}^{2} J_{x}$ in the above equation equal to $\frac{\Delta x^{2}}{8} \lambda^{2}$.

\section{- Accordion test case}

To make a further test of the analysis of boundary conditions, we have chosen the following problem: we consider a rectangular domain of size $\Omega=] 0, L[\times] 0, h[$, with periodic boundary conditions at $x=0$ and $x=L$. For the boundaries at $y=0$ and $y=h$ we have: $J_{x}(x, 0)=J_{x}(x, h)=J_{0} \cos \left(2 k \pi \frac{x}{L}\right), J_{y}(x, 0)=J_{y}(x, h)=0$, $0<x<L$, where $k$ is some integer. In the low velocity regime (easily simulated with the LBE technique by removing non linear terms in the equilibrium values of the non-conserved moments) the steady state is solution of the Stokes equations (3): $\operatorname{div} J=0,-\nu \Delta J+\nabla p=0$. We note here that the accordion test case is incompressible with a constant density in space. The solution is given by a simple analytic expression for a stream function $\psi$ such that

$$
J_{x}=\frac{\partial \psi}{\partial y}, \quad J_{y}=-\frac{\partial \psi}{\partial x}, \quad \psi=f(y) \cos (\mathcal{K} x), \quad \Delta^{2} \psi=0,
$$


with $\Delta^{2}$ the bi-harmonic operator and $\mathcal{K} \equiv \frac{2 k \pi}{L}$. After an elementary calculs, we get

$$
f(y)=a \sinh (\mathcal{K} y)+b y \cosh (\mathcal{K} y)+c y \sinh (\mathcal{K} y)
$$

where $\{a, b, c\}$ are obtained by the boundary condition $f=0$ and $\partial_{y} f=J_{x}$ on the boundaries located at $y=0$ and $y=h$. We get:

$$
a=-h \frac{J_{0}}{\sinh (\mathcal{K} h)-\mathcal{K} h}, \quad b=J_{0} \frac{\sinh (\mathcal{K} h)}{\sinh (\mathcal{K} h)-\mathcal{K} h}, \quad \text { and } \quad c=J_{0} \frac{1-\cosh (\mathcal{K} h)}{\sinh (\mathcal{K} h)-\mathcal{K} h} .
$$

From $f(y)$ one gets the velocity field and when necessary all kinds of spatial derivatives. The pressure can also be obtained as :

$$
p(x, y)=\frac{\nu}{\mathcal{K}} \sin (\mathcal{K} x)\left(\frac{\mathrm{d}^{3} f}{\mathrm{~d} y^{3}}-\mathcal{K}^{2} \frac{\mathrm{d} f}{\mathrm{~d} y}\right),
$$

up to some constant, where $\nu$ is the kinematic shear viscosity. The momentum field $\left(J_{x}, J_{y}\right)$ can be directly compared between the analytic solution and the result of the lattice Boltzmann experiment. In a sense this "elementary situation" can be seen as an extension of the much studied Poiseuille case. It may help to understand a little better what happens in situations where the flow is non uniform along a boundary. Note that as we consider only the linear regime, a more complicated periodic function for the imposed velocities of the boundaries can be treated in the same manner by first decomposing the imposed velocity in Fourier components. The lattice Boltzmann simulation is carried out for a large enough number of time steps so that a steady state is reached. We call $\left\{j_{x}, j_{y}\right\}$ the "experimental" field and $\left\{J_{x}, J_{y}\right\}$ the "theoretical" field obtained as described in the previous sub-section. (These notations are chosen in agreement with those in the Appendix.)

Remark. Lattice Boltzmann scheme is not incompressible (for instance speed of sound is finite). Now we can estimate the errors of $\operatorname{div} j=0$ by finite difference method, using simple 5 -points stencil leads to maximum divergence of $3.10^{-5}$. Using 9-points stencil for the same flow leads to maximum error of $3.10^{-8}$, three order magnitude smaller than 5-points stencil. Note that these results hardly change when we replace finite difference applied to measured flux $j$ or the velocity $j / \rho$. It is observed that the density $\rho$ varies between $1 \pm 7.10^{-4}$ in our accordion test. We verified that each simulation lasts long enough to reach a steady state. Density measurements vary by less than $7.10^{-12}$ in 1000 time steps, that in particular for the density in an edge point and a center point. The analytic field given by (22), (23) and (24) is more useful reference for our LB flow than what we could get from a simple finite difference simulation of a weakly compressible Navier Stokes flow.

We now consider a series of computations with the same aspect ratio for the rectangle $\left(R \equiv N_{y} / N_{x}=\right.$ constant, where $N_{x}$ and $N_{y}$ are respectively the number of nodes in the $x$ and $y$ direction) and determine the root mean square of the difference of the two fields $j$ and $J$ : this allows to verify that we should use the values of the boundary velocity where the lines $f_{5}^{*}$ and $f_{6}^{*}$ cross the numerical boundary as shown in Figure 3.

We also verify that the velocity component $j_{x}$ varies as $\cos (\mathcal{K} x)$ while the velocity component $j_{y}$ varies as $\sin (\mathcal{K} x)$. This allows us to characterize accurately the "experimental" field by just two functions:

$$
g_{x}(y)=\int_{0}^{L} j_{x} \cos (\mathcal{K} x) \mathrm{d} x \quad \text { and } \quad g_{y}(y)=\int_{0}^{L} j_{y} \sin (\mathcal{K} x) \mathrm{d} x
$$

and the same for the "theoretical" field $\left(G_{x}(y)\right.$ and $\left.G_{y}(y)\right)$ with proper normalization such that $G_{x}=1$ on the boundary $y=0$.

Test of equations (18),(20): for a rectangle of aspect ratio $R=\{1,2,4,8\}$ we compare the measured $g_{x}$ and $g_{y}$ on the boundary $y=0$ with successive approximations shown in equations (18),(20) for several values of $N_{y}$ as shown in Figure 4, 5, 6 and 7 . In table 1 we show the convergence rates $\theta$ (error is proportional to $\Delta t^{\theta}$ ) of the equations (18),(20) for aspect ratio $R=\{1,2,4,8\}$. The results show a good agreement with the expected theoretical results given by the equations $(18),(20)$. 


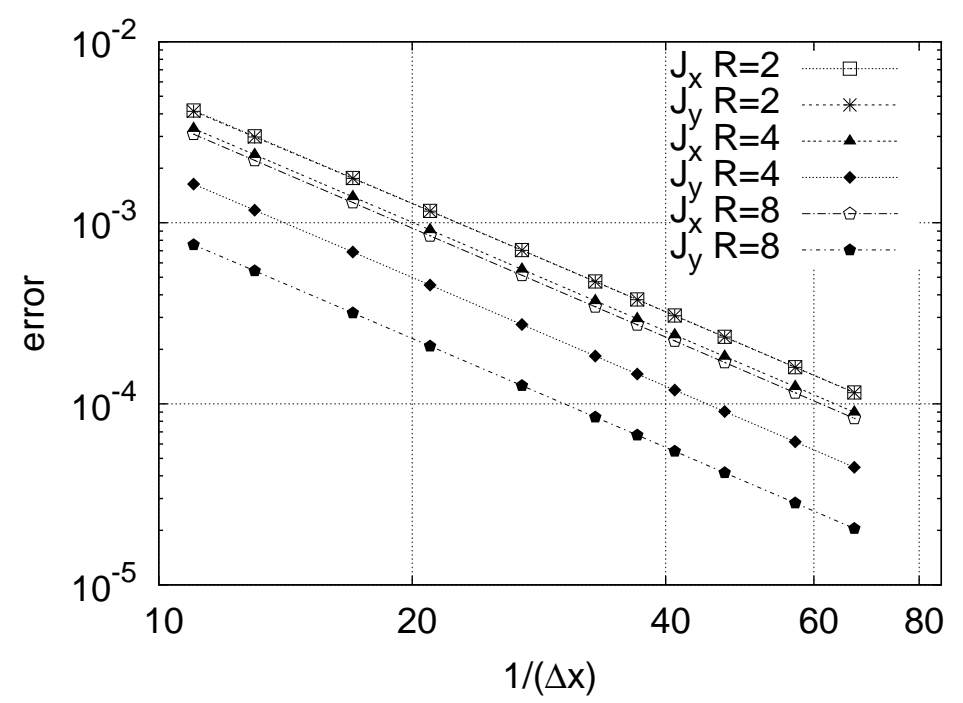

FIGURE 3. Second order convergence for the global domain. The $\ell^{2}$ error between LBE velocity field $\left\{j_{x}, j_{y}\right\}$ defined in (5) and the analytical velocity solution field $\left\{J_{x}, J_{y}\right\}$ is presented for different aspect ratio $R$.

\begin{tabular}{|c|c|c|c||c|c|c|c|}
\hline Ratio & Order & $\left|J_{x}-j_{x}\right|$ & $\left|J_{y}-j_{y}\right|$ & Ratio & Order & $\left|J_{x}-j_{x}\right|$ & $\left|J_{y}-j_{y}\right|$ \\
\hline 1 & 1 & 0.903 & 0.807 & 2 & 1 & 0.950 & 0.883 \\
\hline & 2 & 1.988 & 1.944 & & 2 & 2.006 & 1.972 \\
\hline & 3 & 2.935 & 4.022 & & 3 & 2.961 & 3.694 \\
\hline \hline 4 & 1 & 0.967 & 0.905 & 8 & 1 & 0.972 & 0.910 \\
\hline & 2 & 2.014 & 1.980 & & 2 & 2.017 & 1.983 \\
\hline & 3 & 2.974 & 3.290 & & 3 & 2.976 & 3.174 \\
\hline
\end{tabular}

TABLE 1. Theoretical and measured rates of convergence $\theta$ (error is proportional to $\Delta t^{\theta}$ ) in the $\ell^{2}$ norm for $x$ and $y$ component of the velocity, for aspect ratio $R=1,2,4,8$.

For the transverse profile, the Figure 8 shows the difference between the measured mean profile $\left(g_{x}(y), g_{y}(y)\right)$ and the theoretical one $\left(G_{x}(y), G_{y}(y)\right)$ for several values of the product $\sigma_{4} \sigma_{7}$. We observe that for this test case the particular value $\sigma_{4} \sigma_{7}=\frac{3}{8}$ does not play a major role for minimizing the boundary error. This remark can be extended to the global error, in fact we see on Figure 9 that the particular value $\sigma_{4} \sigma_{7}=\frac{3}{8}$ has no direct influence for the minimization of the global error in the whole domain.

\section{TRANSFER OF LINEAR MOMENTUM}

We put in evidence the viscous stress (i.e. wall friction) $\tau$ plus additional terms of higher order associated to second order derivatives of the velocity field. These terms are estimated with finite differences. We test the previous ideas for a Poiseuille flow and an "accelerated pipe" defined as follows. On the left (resp. right) boundary, a given pressure $+P$ (resp $-P$ ) is imposed. On bottom and top boundaries, a given velocity is null for $x \leq-L$ and for $x \geq+L$. It is a smooth increasing function for $-L \leq x \leq 0$ and a smooth decreasing function for $0 \leq x \leq L$. The previous study has been also applied to the computation of pressure on a wall and the adaptation of anti-bounce-back boundary condition for a precise implementation of a given pressure 
boundary condition. The aim is to estimate the wall friction (parallel momentum exchange) using the above analysis of the bounce-back. On boundary point $x_{b}$ the change in momentum due to the wall is composed by two terms: $f_{8}^{*}\left(x_{l}\right)-f_{7}^{*}\left(x_{r}\right)$ for the incoming particles and $f_{5}^{*}\left(o_{l}\right)-f_{6}^{*}\left(o_{r}\right)$ for the outgoing particles, as presented in Figure 10. In consequence, we propose to evaluate the transfer of moments by the following formula:

$$
T_{C} \equiv\left[f_{8}^{*}\left(x_{l}\right)-f_{7}^{*}\left(x_{r}\right)\right]-\left[f_{5}^{*}\left(o_{l}\right)-f_{6}^{*}\left(o_{r}\right)\right] .
$$

Due to the bounce-back algorithm, the momentum transfer is

$$
T_{C}=2\left[f_{8}^{*}\left(x_{l}\right)-f_{7}^{*}\left(x_{r}\right)\right]+\text { given boundary terms } .
$$

We note that Ladd [16] and Mei et al. [20] have used the "cross" scheme for momentum transfer to evaluate the force on the wall. Here, we use the relation (25) to estimate numerically the friction $\tau \equiv \mu\left(\partial_{y} u_{x}+\partial_{x} u_{y}\right)$ parallel to the boundary, where the shear viscosity is given by the relation $\nu=\frac{\sigma_{4}}{6} \lambda^{2} \Delta t$. We also give a development of the relation (25) up to order two.

- Proposition 5. "Cross" stencil. Using the Taylor development of the moment on the wall $x$ up to order two, with the bounce-back boundary condition, we have:

$$
\left\{\begin{aligned}
T_{C}=\frac{1}{3 \lambda} J_{x}+ & \tau-\frac{\Delta t}{\lambda}\left(\frac{1}{2}+\sigma_{4}\right)\left(\partial_{t} J_{x}+c_{0}^{2} \partial_{x} \rho\right)+\frac{\Delta t}{6 \lambda} \partial_{t} J_{x} \\
+\Delta t^{2} & \left(\theta_{t t} \partial_{t}^{2} J_{x}+\theta_{t y} \partial_{t} \partial_{y} J_{x}+\theta_{x x} \partial_{x}^{2} J_{x}+\theta_{y y} \partial_{y}^{2} J_{x}\right. \\
& \left.+\beta_{t x} \partial_{t} \partial_{x} J_{y}+\beta_{x y} \partial_{x} \partial_{y} J_{y}+\kappa_{t x} \partial_{t} \partial_{x} \rho+\kappa_{x y} \partial_{x} \partial_{y} \rho\right)+\mathrm{O}\left(\Delta t^{3}\right)
\end{aligned}\right.
$$

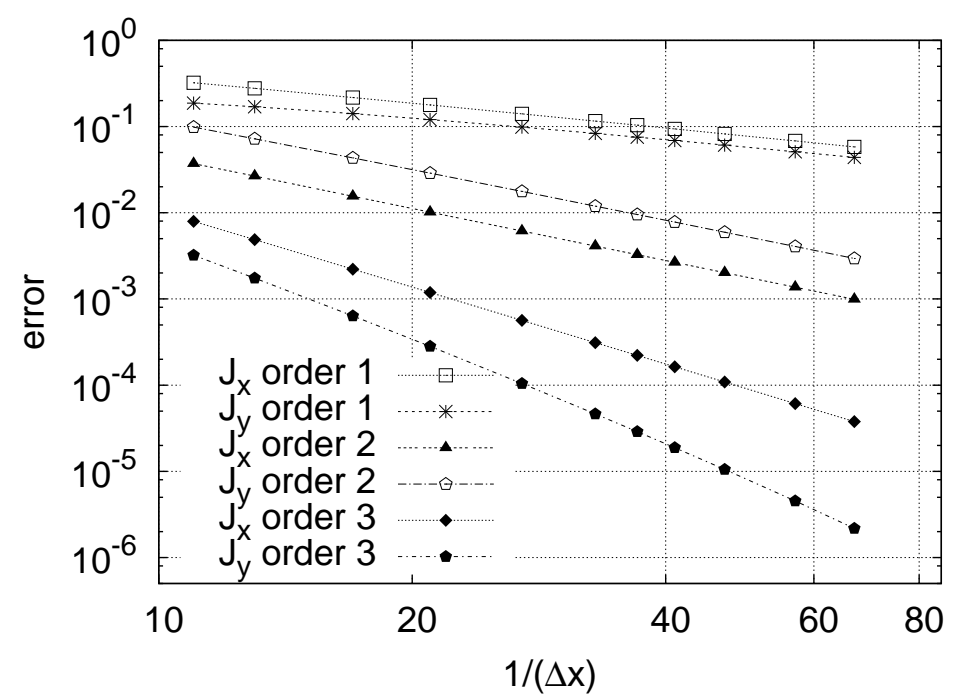

FiguRE 4. Difference of measurement and successive approximations in equations (18), (20), for a rectangle of aspect ratio $R=1$. 


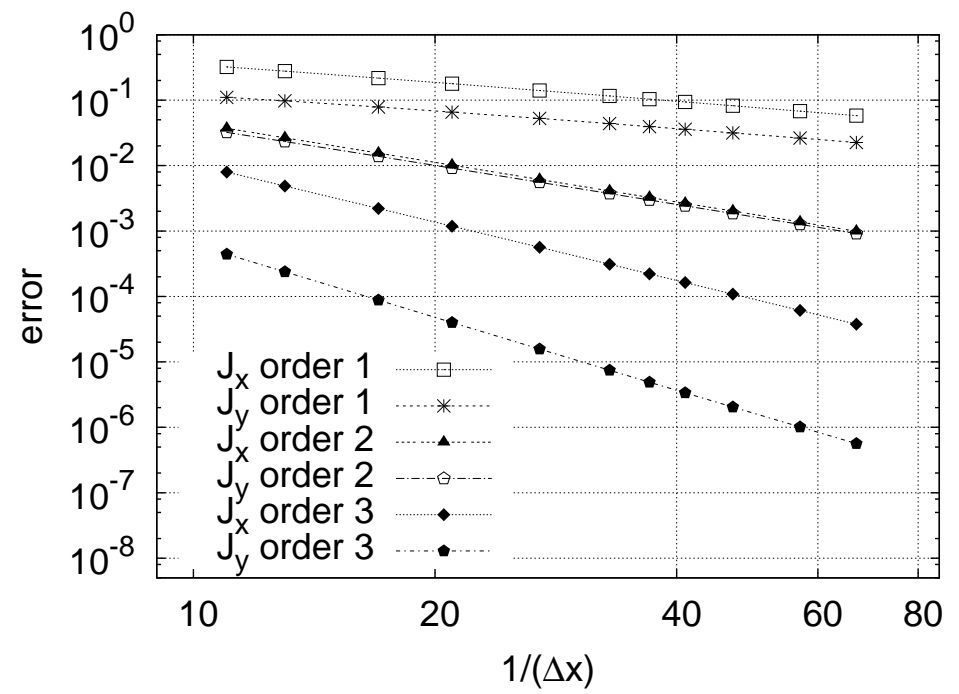

FiguRE 5. Difference of measurement and successive approximations in equations (18), (20), for a rectangle of aspect ratio $R=2$

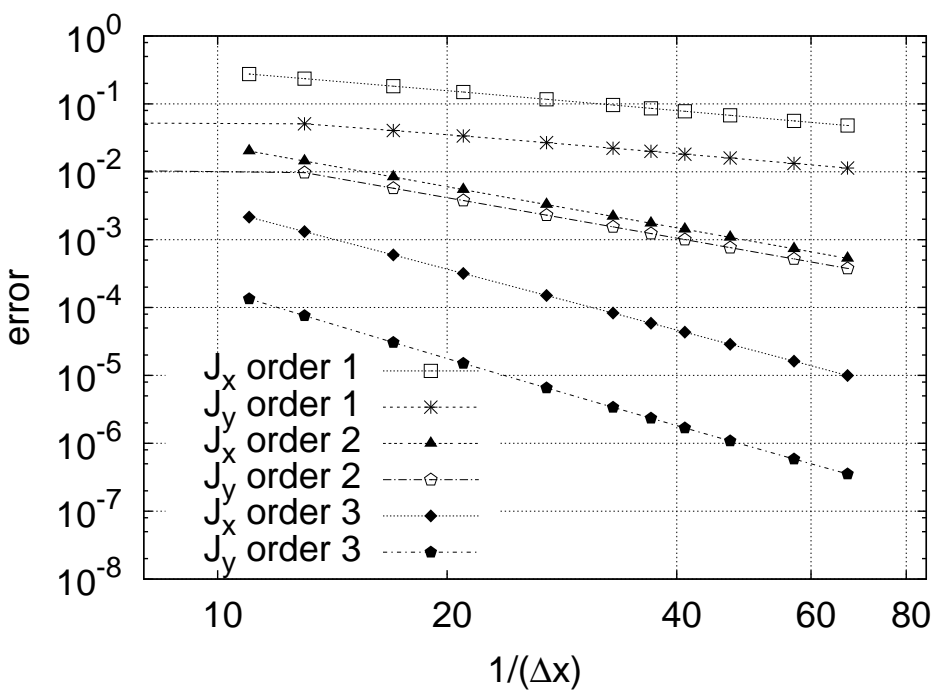

FigurE 6. Difference of measurement and successive approximations in equations (18), (20), for a rectangle of aspect ratio $R=4$.

where $c_{0}^{2}=\frac{\lambda^{2}}{3}\left(a_{2}+4\right)$ is the sound speed and

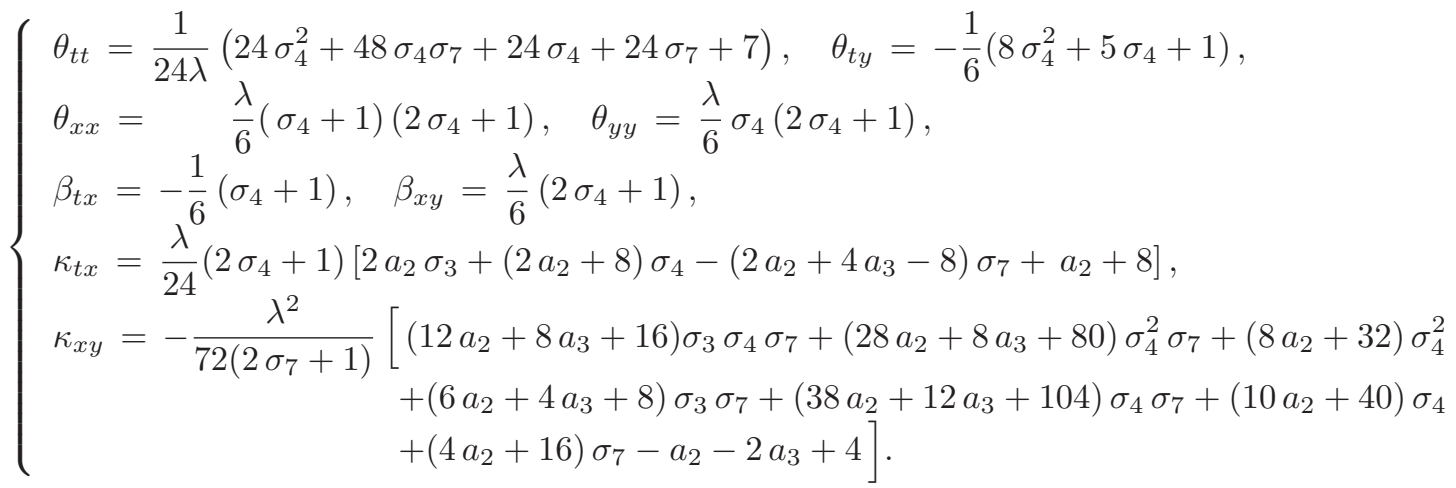


- With the hat stencil of Figure 11, the momentum transfer can be approximated by the relation

$$
T_{H}\left(x_{i}, t\right) \equiv 2\left[f_{8}^{*}\left(x_{i}, t\right)-f_{7}^{*}\left(x_{i}, t\right)\right]+\text { given boundary terms. }
$$

We have the following

\section{- Proposition 6. "Hat" stencil.}

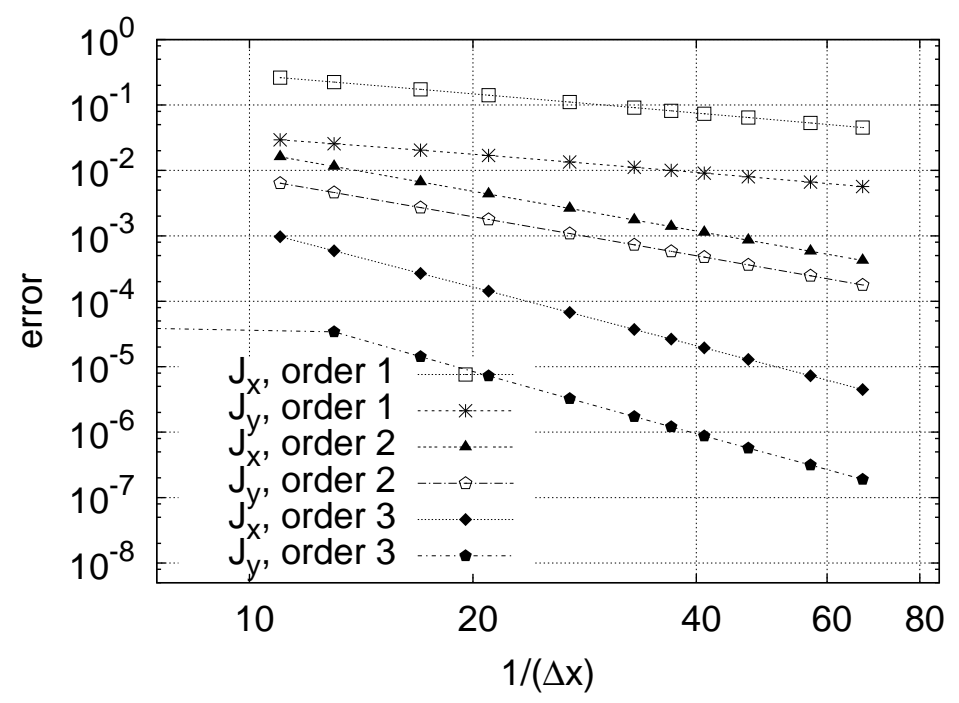

FiguRE 7. Difference of measurement and successive approximations in equations (18), (20), for a rectangle of aspect ratio $R=8$.
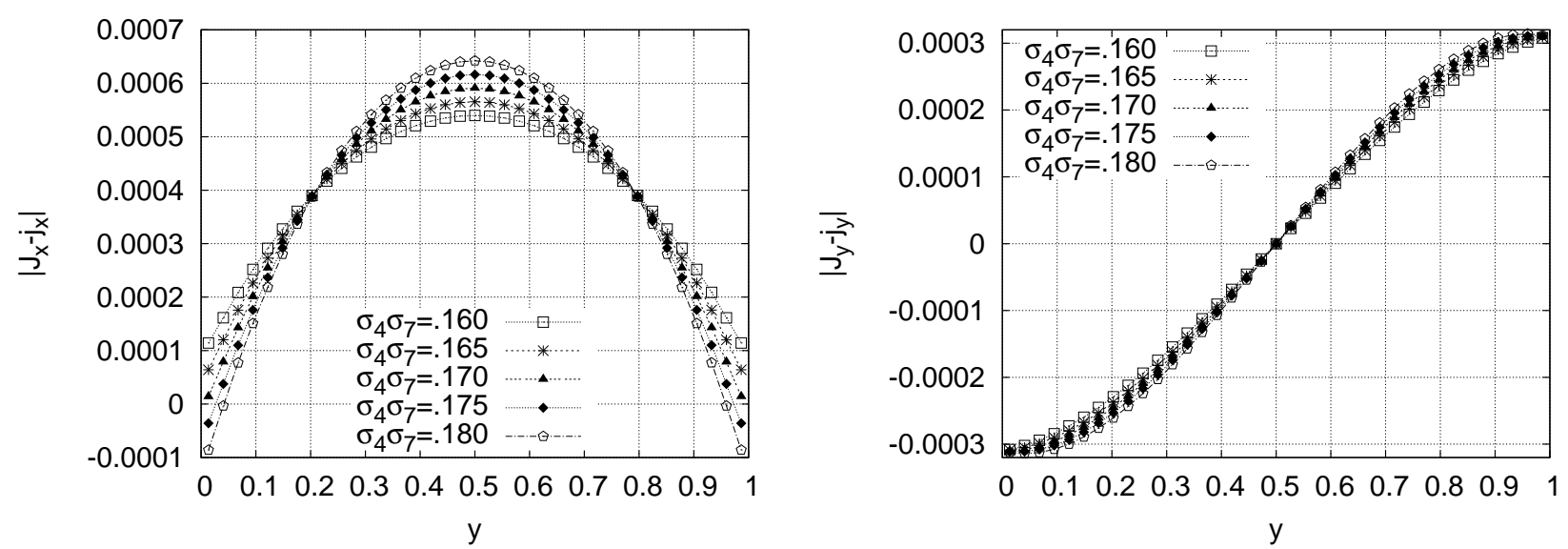

FiguRE 8. Difference of measured and theoretical profile for several values of the product $\sigma_{4} \sigma_{7}$. (left) for $x$-velocity and (right) for the $y$-velocity. The value of $\sigma_{4} \sigma_{7}$ that minimize the error at the boundary is near 0.17 , and this value is not the one $(3 / 8=0.375)$ predicted by Poiseuille like analysis. 

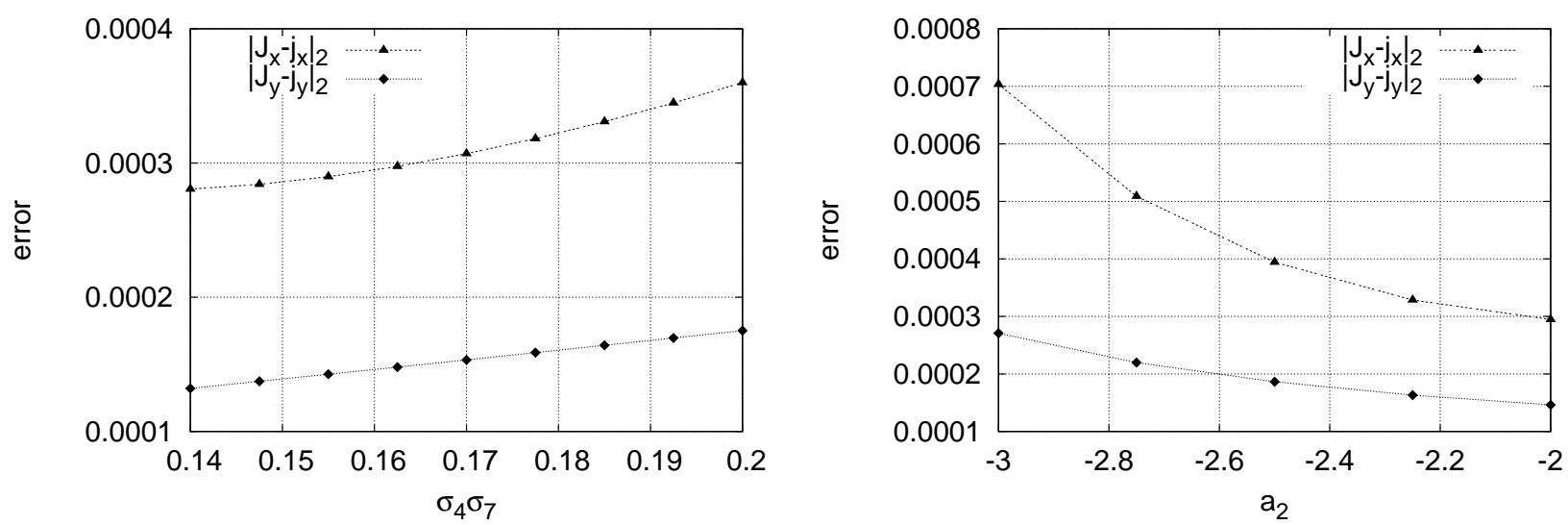

FiguRE 9. Profile of the error of the $x$ and $y$ component of the velocity for $N_{y}=37$ and $R=4$, (left) versus the product $\sigma_{4} \sigma_{7}$ and (right) versus the coefficient $a_{2}$.

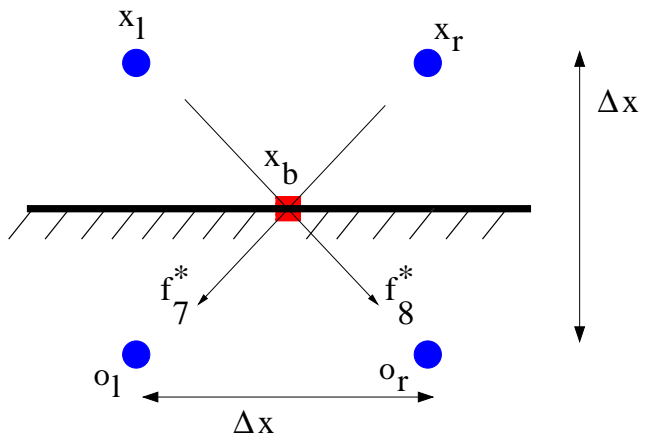

Figure 10. Discrete computation of the wall friction with a "cross" scheme.

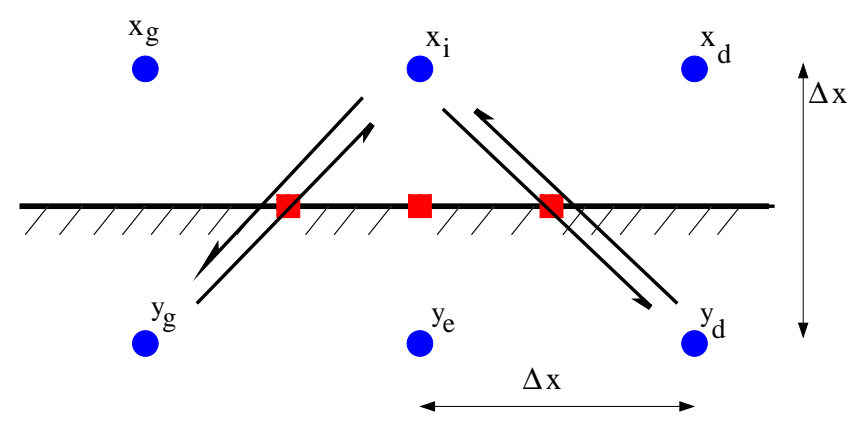

FiguRE 11. Discrete computation of the wall friction with a "hat" scheme.

Using the Taylor development of the moment on the wall $x$ up to order two, with the bounce-back boundary condition, we have:

$$
\left\{\begin{aligned}
T_{H}=\frac{1}{3 \lambda} J_{x}+\tau & -\frac{\Delta t \sigma_{4}}{\lambda}\left(\partial_{t} J_{x}+c_{0}^{2} \partial_{x} \rho\right)+\frac{\Delta t}{2 \lambda} \partial_{t} J_{x}+\frac{\lambda}{36}\left(7 a_{2}+2 a_{3}+20\right) \partial_{x} \rho \\
+\Delta t^{2} & \left(\widetilde{\theta}_{t t} \partial_{t}^{2} J_{x}+\widetilde{\theta}_{t y} \partial_{t} \partial_{y} J_{x}+\widetilde{\theta}_{x x} \partial_{x}^{2} J_{x}+\widetilde{\theta}_{y y} \partial_{y}^{2} J_{x}\right. \\
+ & \left.\widetilde{\beta}_{t x} \partial_{t} \partial_{x} J_{y}+\widetilde{\beta}_{x y} \partial_{x} \partial_{y} J_{y}+\widetilde{\kappa}_{t x} \partial_{t} \partial_{x} \rho+\widetilde{\kappa}_{x y} \partial_{x} \partial_{y} \rho\right)+\mathrm{O}\left(\Delta t^{3}\right)
\end{aligned}\right.
$$


where

$$
\left\{\begin{array}{l}
\tilde{\theta}_{t t}=\frac{1}{4 \lambda}\left(2 \sigma_{4}-1\right)\left(2 \sigma_{4}+4 \sigma_{7}+3\right), \quad \tilde{\theta}_{t y}=-\frac{1}{12}\left(16 \sigma_{4}^{2}+4 \sigma_{4}-1\right) \\
\tilde{\theta}_{x x}=\quad \frac{\lambda}{18}\left(6 \sigma_{4}^{2}+3 \sigma_{4}+\sigma_{8}-5\right), \quad \tilde{\theta}_{y y}=\frac{\lambda}{3} \sigma_{4}^{2} \\
\widetilde{\beta}_{t x}=-\frac{1}{36}\left(18 \sigma_{4}+2 \sigma_{8}-7\right), \quad \widetilde{\beta}_{x y}=\frac{\lambda}{18}\left(9 \sigma_{4}+\sigma_{8}-5\right) \\
\widetilde{\kappa}_{t x}=\frac{\lambda}{72}\left(12 a_{2} \sigma_{3} \sigma_{4}+12 a_{2} \sigma_{4}^{2}+48 \sigma_{4}^{2}-12 a_{2} \sigma_{4} \sigma_{7}-24 a_{3} \sigma_{4} \sigma_{7}+48 \sigma_{4} \sigma_{7}\right. \\
\left.-14 a_{2} \sigma_{3}+12 a_{2} \sigma_{4}+72 \sigma_{4}+6 a_{2} \sigma_{7}+12 a_{3} \sigma_{7}-24 \sigma_{7}-4 a_{3} \sigma_{8}-5 a_{2}+2 a_{3}-48\right) \\
\quad\left[\left(36 a_{2}+24 a_{3}+48\right) \sigma_{3} \sigma_{4} \sigma_{7}+\left(84 a_{2}+24 a_{3}+240\right) \sigma_{4}^{2} \sigma_{7}+\left(24 a_{2}+96\right) \sigma_{4}^{2}\right. \\
-\left(42 a_{2}+28 a_{3}+56\right) \sigma_{3} \sigma_{7}+\left(66 a_{2}+12 a_{3}+216\right) \sigma_{4} \sigma_{7}-8\left(a_{2}+a_{3}\right) \sigma_{7} \sigma_{8} \\
\left.+\left(24 a_{2}+96\right) \sigma_{4}+\left(22 a_{2}+12 a_{3}+40\right) \sigma_{7}+\left(8+2 a_{2}\right) \sigma_{8}-7 a_{2}-6 a_{3}-4\right]
\end{array}\right.
$$

Remark. For the Poiseuille problem with the bounce-back boundary condition the above formula becomes:

$$
T_{H}\left(x_{i}, t\right)=\Delta t \sigma_{4} \frac{\lambda}{3} \frac{\partial J_{y}}{\partial x}+\Delta t^{2}\left[\sigma_{4} \frac{\lambda^{2}}{9} \frac{4+2 a_{2}+a_{3}}{4+a_{2}} \frac{\partial^{2} J_{y}}{\partial x^{2}}\right]+\mathrm{O}\left(\Delta t^{3}\right) .
$$

\section{- Numerical results for cross stencil in the case of Poiseuille flow}

We test here the $T_{H}$ for Poiseuille problem. Let $\Omega=\left[1, N_{x}\right] \times\left[1, N_{y}\right]$ we take the following boundary conditions: Anti-Bounce-back boundary condition on (inlet) $i=1$ and (outlet) $i=N_{x}$ to impose $p= \pm \delta_{p}$. Bounce-back boundary condition on: $j=1$ and $j=N_{y}$ to impose $j_{x}=0$. Figure 12 shows $T_{H}$ and $\tau$ versus space step $\Delta x$ for two different values of $a_{2}$ and $a_{3}$. For both case we see that the convergence is order 2 . In Figure 13 we show differences between the quantities (26) and (27) versus space step $\Delta x$. For both cases of values of $a_{2}$ and $a_{3}$ we get machine accuracy.

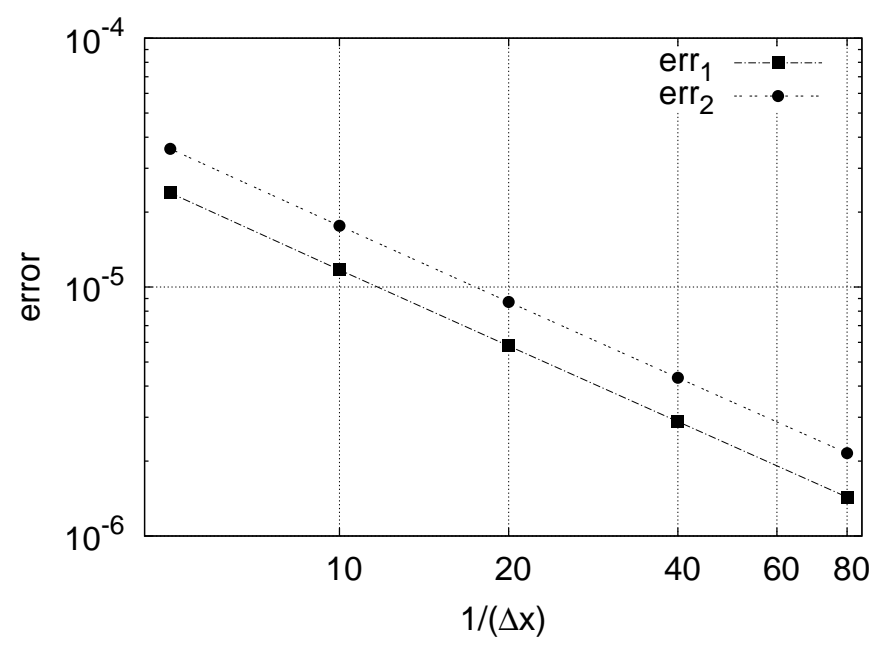

Figure 12. Difference between the $T_{H}=2\left[f_{8}^{*}\left(x_{i}\right)-f_{7}^{*}\left(x_{i}\right)\right]$ and wall friction $\tau$ versus $\Delta x$. The case $\square$ is when $a_{2}=-2$ and $a_{3}=1$, second case $\bullet$ is when $a_{2}=-2.5$ and $a_{3}=2.5$ 


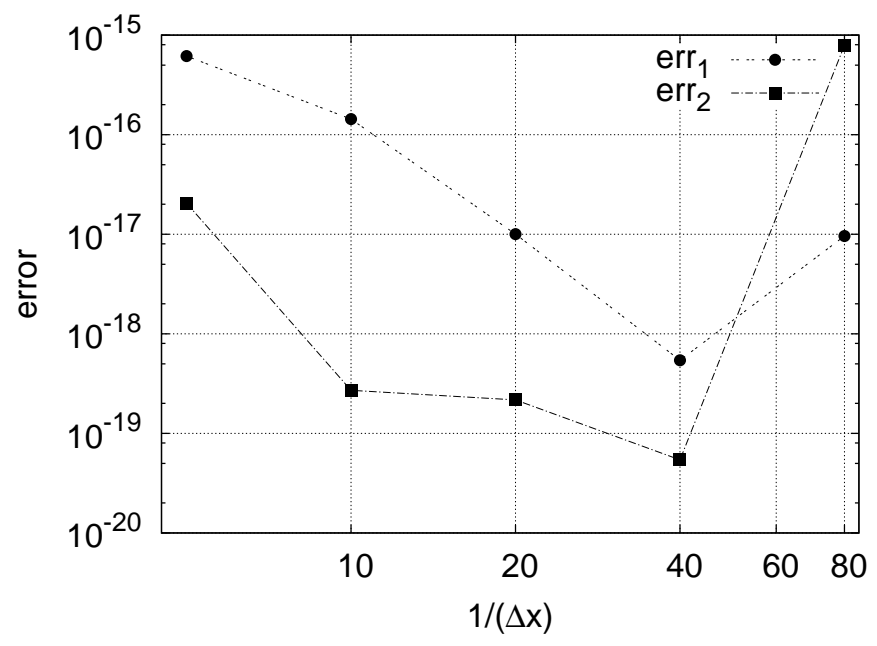

FiguRE 13. Difference between the $T_{H}=2\left[f_{8}^{*}\left(x_{i}\right)-f_{7}^{*}\left(x_{i}\right)\right]$ and $T$ given by the above proposition versus $\Delta x$. The case $\square$ is when $a_{2}=-2$ and $a_{3}=1$, second case $\bullet$ is when $a_{2}=-2.5$ and $a_{3}=2.5$.

\section{CONCLUSiOn}

We have proposed a formal method for the analysis of a bounce-back boundary condition for the lattice Boltzmann scheme. The present approach is an extension of the Taylor expansion method. We have also given a precise development of the wall friction via parallel momentum exchange. Our theoretical results are validated not only for the linear Poiseuille flow but also for accordion flow. We have analytical solutions for both tests. The numerical results show good agreement at first and second order accuracy. A specific observation for the accordion flow concerns specific values of the MRT parameters. Specific values lead to machine accuracy for the Poiseuille flow, but we observe for the more difficult accordion test case good convergence properties not directly related to special values of the parameters $\sigma_{4} \sigma_{7}$. In future work we will extend this study to orthogonal momentum exchange, study the link with pressure and anti-bounce-back boundary conditions, study time dependent boundary conditions, consider non linear effects for the LB scheme and finally give more general boundary condition for any position of the wall in the lattice. In particular we wish to present both linear and nonlinear momentum transfer for the accordion case in a coming further paper.

\section{ACKNOWLEGMENT}

We gratefully thank the two referees for their useful remarks. Some of them were directly integrated in the final version of the manuscript. 


\section{AppendiX Relative to The D2Q9 scheme}

For the D2Q9 lattice Boltzmann scheme, we introduce a constant velocity ratio $\lambda$ between space step and time step and we use the following matrix $M$ for the transformation of the particle distribution into moments:

$$
M=\left(\begin{array}{ccccccccc}
1 & 1 & 1 & 1 & 1 & 1 & 1 & 1 & 1 \\
0 & \lambda & 0 & -\lambda & 0 & \lambda & -\lambda & -\lambda & \lambda \\
0 & 0 & \lambda & 0 & -\lambda & \lambda & \lambda & -\lambda & -\lambda \\
-4 \lambda^{2} & -\lambda^{2} & -\lambda^{2} & -\lambda^{2} & -\lambda^{2} & 2 \lambda^{2} & 2 \lambda^{2} & 2 \lambda^{2} & 2 \lambda^{2} \\
0 & \lambda^{2} & -\lambda^{2} & \lambda^{2} & -\lambda^{2} & 0 & 0 & 0 & 0 \\
0 & 0 & 0 & 0 & 0 & \lambda^{2} & -\lambda^{2} & \lambda^{2} & -\lambda^{2} \\
0 & -2 \lambda^{3} & 0 & 2 \lambda^{3} & 0 & \lambda^{3} & -\lambda^{3} & -\lambda^{3} & \lambda^{3} \\
0 & 0 & -2 \lambda^{3} & 0 & 2 \lambda^{3} & \lambda^{3} & \lambda^{3} & -\lambda^{3} & -\lambda^{3} \\
4 \lambda^{4} & -2 \lambda^{4} & -2 \lambda^{4} & -2 \lambda^{4} & -2 \lambda^{4} & \lambda^{4} & \lambda^{4} & \lambda^{4} & \lambda^{4}
\end{array}\right) .
$$

The moments $m$ are classically [18] named as follows

$$
m=\left(\rho, j_{x}, j_{y}, e, x x, x y, q_{x}, q_{y}, e_{2}\right)^{\mathrm{t}} .
$$

In this contribution, the equilibrium values are supposed to be linear functions of the conserved moments $W \equiv\left(\rho, j_{x}, j_{y}\right)$ :

$$
m^{\mathrm{eq}} \equiv\left(\rho, j_{x}, j_{y}, e^{\mathrm{eq}}=a_{2} \lambda^{2} \rho, x x^{\mathrm{eq}}=0, x y^{\mathrm{eq}}=0, q_{x}^{\mathrm{eq}}=-\lambda^{2} j_{x}, q_{y}^{\mathrm{eq}}=-\lambda^{2} j_{y}, e_{2}^{\mathrm{eq}}=\lambda^{4} a_{3} \rho\right)^{\mathrm{t}} .
$$

The parameter $a_{2}$ is related to the sound speed $c_{0}=\sqrt{\frac{4+a_{2}}{6}} \lambda$ and parameter $a_{3}$ is adjustable parameter without any effect in equivalent equation up to order two (see [18] for more details). The relaxation matrix $S$ is given as follows :

$$
S=\operatorname{diag}\left(0,0,0, s_{3}, s_{4}, s_{4}, s_{7}, s_{7}, s_{8}\right) \text {. }
$$

If we associate the relaxation (2) with the previous equilibria (29), we can write globally $m^{*}=J_{0} m$ and the matrix $J_{0}$ admits the expression

$$
J_{0}=\left(\begin{array}{ccccccccc}
1 & 0 & 0 & 0 & 0 & 0 & 0 & 0 & 0 \\
0 & 1 & 0 & 0 & 0 & 0 & 0 & 0 & 0 \\
0 & 0 & 1 & 0 & 0 & 0 & 0 & 0 & 0 \\
a_{2} s_{3} \lambda^{2} & 0 & 0 & 1-s_{3} & 0 & 0 & 0 & 0 & 0 \\
0 & 0 & 0 & 0 & 1-s_{4} & 0 & 0 & 0 & 0 \\
0 & 0 & 0 & 0 & 0 & 1-s_{4} & 0 & 0 & 0 \\
0 & -s_{7} \lambda^{2} & 0 & 0 & 0 & 0 & 1-s_{7} & 0 & 0 \\
0 & 0 & -s_{7} \lambda^{2} & 0 & 0 & 0 & 0 & 1-s_{7} & 0 \\
a_{3} s_{8} \lambda^{4} & 0 & 0 & 0 & 0 & 0 & 0 & 0 & 1-s_{8}
\end{array}\right) .
$$

For the bottom boundary condition described at the Figure 2, the matrix $T$ is simply given by

$$
T=\left(\begin{array}{lllllllll}
0 & 0 & 0 & 0 & 0 & 0 & 0 & 0 & 0 \\
0 & 0 & 0 & 0 & 0 & 0 & 0 & 0 & 0 \\
0 & 0 & 0 & 0 & 1 & 0 & 0 & 0 & 0 \\
0 & 0 & 0 & 0 & 0 & 0 & 0 & 0 & 0 \\
0 & 0 & 0 & 0 & 0 & 0 & 0 & 0 & 0 \\
0 & 0 & 0 & 0 & 0 & 0 & 0 & 1 & 0 \\
0 & 0 & 0 & 0 & 0 & 0 & 0 & 0 & 1 \\
0 & 0 & 0 & 0 & 0 & 0 & 0 & 0 & 0 \\
0 & 0 & 0 & 0 & 0 & 0 & 0 & 0 & 0
\end{array}\right)
$$


- The matrix $K$ introduced at the relation (10) takes the form

$$
K=\left(\begin{array}{ccccccccc}
0 & 0 & \frac{1}{\lambda} & 0 & 0 & 0 & 0 & 0 & 0 \\
0 & \frac{2}{3}-\frac{s_{7}}{3} & 0 & 0 & 0 & 0 & \frac{1-s_{7}}{3 \lambda^{2}} & 0 & 0 \\
0 & 0 & 1 & 0 & 0 & 0 & 0 & 0 & 0 \\
-s_{3} a_{2} \lambda^{2} & 0 & \lambda\left(1-s_{7}\right) & s_{3} & 0 & 0 & 0 & \frac{1-s_{7}}{\lambda} & 0 \\
0 & 0 & -\frac{\lambda}{3}\left(1+s_{7}\right) & 0 & s_{4} & 0 & 0 & \frac{1-s_{7}}{3 \lambda} & 0 \\
0 & \frac{\lambda}{3}\left(2-s_{7}\right) & 0 & 0 & 0 & s_{4} & \frac{1-s_{7}}{3 \lambda} & 0 & 0 \\
0 & \frac{2}{3} \lambda^{2}\left(1+s_{7}\right) & 0 & 0 & 0 & 0 & \frac{1}{3}+\frac{2 s_{7}}{3} & 0 & 0 \\
0 & 0 & 0 & 0 & 0 & 0 & 0 & 1 & 0 \\
-a_{3} s_{8} \lambda^{4} & 0 & -s_{7} \lambda^{3} & 0 & 0 & 0 & 0 & \lambda\left(1-s_{7}\right) & s_{8}
\end{array}\right) .
$$

We consider a given vector $g \equiv\left(g_{0}, \ldots, g_{8}\right) \in \mathbb{R}^{9}$. The matrix $K$ is singular and holds a kernel of dimension 1 . In fact the third row is a multiple of the first row. Then when we solve a linear system of the type

$$
K \mu=g,
$$

the right hand side has to satisfy the compatibility relation

$$
g_{2}-\lambda g_{0}=0 .
$$

Then the solution of (33) admits the expression

$$
\mu=\rho \mu_{0}+\Sigma g
$$

where

$$
\mu_{0} \equiv\left(1,0,0, a_{2} \lambda^{2}, 0,0,0,0, a_{3} \lambda^{4}\right)^{\mathrm{t}}
$$

is a generator of the kernel of the matrix $K$. The parameter $\rho$ appears as some integration constant and the matrix $\Sigma$ is given by the expression

$$
\Sigma=\left(\begin{array}{ccccccccc}
0 & 0 & 0 & 0 & 0 & 0 & 0 & 0 & 0 \\
0 & 2+\frac{1}{s_{7}} & 0 & 0 & 0 & 0 & \frac{1}{\lambda^{2}}\left(1-\frac{1}{s_{7}}\right) & 0 & 0 \\
0 & 0 & 1 & 0 & 0 & 0 & 0 & 0 & 0 \\
0 & 0 & \frac{\lambda}{s_{3}}\left(s_{7}-1\right) & \frac{1}{s_{3}} & 0 & 0 & 0 & \frac{1}{\lambda s_{3}}\left(s_{7}-1\right) & 0 \\
0 & 0 & \frac{\lambda}{3 s_{4}}\left(1+s_{7}\right) & 0 & \frac{1}{s_{4}} & 0 & 0 & \frac{s_{7}-1}{3 \lambda s_{4}} & 0 \\
0 & -\frac{\lambda}{s_{4}} & 0 & 0 & 0 & \frac{1}{s_{4}} & 0 & 0 & 0 \\
0 & -2 \lambda^{2}\left(1+\frac{1}{s_{7}}\right) & 0 & 0 & 0 & 0 & \frac{2}{s_{7}}-1 & 0 & 0 \\
0 & 0 & 0 & 0 & 0 & 0 & 0 & 1 & 0 \\
0 & 0 & \lambda^{3} \frac{s_{7}}{s_{8}} & 0 & 0 & 0 & 0 & \frac{\lambda}{s_{8}}\left(s_{7}-1\right) & \frac{1}{s_{8}}
\end{array}\right) .
$$

- The solution of the linear system (11) at order zero is easy to made explicit for the D2Q9 scheme. First, due to the bounce-back (6) boundary conditions, the right hand side $M \xi$ takes the form

$$
M \xi_{0}=\left(\rho, J_{x}, J_{y}, a_{2} \lambda^{2} \rho, 0,0,-\lambda^{2} J_{x},-\lambda^{2} J_{y}, \lambda^{4} a_{3} \rho\right)^{\mathrm{t}} .
$$


The compatibility condition (34) is trivial for the right hand side (36). Then the relation (35) simply gives

$$
m^{0}=\left(\rho, J_{x}, J_{y}, a_{2} \lambda^{2} \rho, 0,0,-\lambda^{2} J_{x},-\lambda^{2} J_{y}, \lambda^{4} a_{3} \rho\right)^{\mathrm{t}} .
$$

It is formally the equilibrium (29), except that we have replaced the momenta $j_{x}$ and $j_{y}$ defined in (5) by the boundary data $J_{x}, J_{y}$. Moreover, the density $\rho$ has still to be determined.

- With the data $\xi$ specified in the right hand side of the relation (6), we have

$$
\xi^{1}=\left\{\begin{array}{r}
\left(\frac{3 \partial_{t} J_{y}+\lambda \partial_{x} J_{x}}{6 \lambda}, \frac{\partial_{t} J_{x}-\lambda \partial_{x} J_{y}}{6}, \frac{3 \partial_{t} J_{y}-\lambda \partial_{x} J_{x}}{6 \lambda},-\frac{\lambda^{2} \partial_{x} J_{x}}{3},-\frac{\lambda \partial_{t} J_{y}}{3}, \frac{\lambda\left(\partial_{t} J_{x}-\lambda \partial_{x} J_{y}\right)}{6},\right. \\
\left.\frac{\lambda^{2}\left(\partial_{t} J_{x}-\lambda \partial_{x} J_{y}\right)}{6},-\frac{\lambda^{2}\left(3 \partial_{t} J_{y}+\lambda \partial_{x} J_{x}\right)}{6},-\frac{\lambda^{2}\left(3 \partial_{t} J_{y}+\lambda \partial_{x} J_{x}\right)}{6}\right)^{\mathrm{t}} .
\end{array}\right.
$$

The matrices $B^{\alpha}$ introduced in (12) take the following expressions for the previous bounce-back problem with the D2Q9 scheme:

$$
\begin{aligned}
& B^{1}=\left(\begin{array}{ccccccccc}
0 & \frac{4+s_{7}}{6} & 0 & 0 & 0 & \frac{1-s_{4}}{2 \lambda} & \frac{\lambda^{2}\left(s_{7}-1\right)}{6} & 0 & 0 \\
\frac{\lambda^{2}\left(8+a_{2} s_{3}-a_{3} s_{8}\right)}{18} & 0 & \frac{\lambda\left(s_{7}-2\right)}{6} & \frac{1-s_{3}}{18} & \frac{1-s_{4}}{2} & 0 & 0 & \frac{\lambda\left(s_{7}-1\right)}{6} & \frac{s_{8}-1}{18 \lambda^{2}} \\
0 & \frac{\lambda\left(s_{7}-2\right)}{6} & 0 & 0 & 0 & \frac{1-s_{4}}{2} & \frac{\lambda^{2}\left(s_{7}-1\right)}{6} & 0 & 0 \\
0 & \frac{\lambda^{2}\left(1-s_{7}\right)}{3} & 0 & 0 & 0 & \lambda\left(s_{4}-1\right) & \frac{2\left(s_{7}-1\right)}{3} & 0 & 0 \\
0 & \frac{\lambda^{2}\left(1+s_{7}\right)}{3} & 0 & 0 & 0 & 0 & \frac{\left(s_{7}-1\right)}{3} & 0 & 0 \\
-\frac{\lambda^{3}\left(4+2 a_{2} s_{3}+a_{3} s_{8}\right)}{18} & 0 & \frac{\lambda\left(2-s_{7}\right)}{6} & \frac{\lambda\left(s_{3}-1\right)}{9} & 0 & 0 & 0 & \frac{s_{7}-1}{6} & \frac{s_{8}-1}{18 \lambda} \\
\frac{\lambda^{4}\left(-4+4 a_{2} s_{3}+5 a_{3} s_{8}\right)}{18} & 0 & \frac{\lambda^{3}\left(s_{7}-2\right)}{6} & \frac{2 \lambda^{2}\left(1-s_{3}\right)}{9} & \lambda^{2}\left(s_{4}-1\right) & 0 & 0 & \frac{\lambda\left(s_{7}-1\right)}{6} & \frac{5\left(s_{8}-1\right)}{18} \\
0 & \frac{\lambda^{3}\left(s_{7}-2\right)}{6} & 0 & 0 & 0 & \frac{\lambda^{2}\left(1-s_{4}\right)}{2} & \frac{\lambda\left(s_{7}-1\right)}{6} & 0 & 0 \\
0 & -\frac{\lambda^{4}\left(2+5 s_{7}\right)}{6} & 0 & 0 & 0 & \frac{\lambda^{3}\left(s_{4}-1\right)}{2} & \frac{5 \lambda\left(s_{7}-1\right)}{6} & 0 & 0
\end{array}\right) \\
& B^{2}=\left(\begin{array}{ccccccccc}
-\frac{\lambda\left(4+a_{2} s_{3}\right)}{12} & 0 & \frac{1}{2} & \frac{s_{3}-1}{12 \lambda} & \frac{s_{4}-1}{4 \lambda} & 0 & 0 & 0 & 0 \\
0 & \frac{\lambda\left(s_{7}-2\right)}{6} & 0 & 0 & 0 & \frac{s_{4}-1}{2} & \frac{s_{7}-1}{6 \lambda} & 0 & 0 \\
\frac{\lambda^{2}\left(4+a_{2} s_{3}\right)}{12} & 0 & -\frac{\lambda}{2} & \frac{s_{3}-1}{12} & \frac{s_{4}-1}{4} & 0 & 0 & 0 & 0 \\
-\frac{\lambda^{3}\left(4+3 a_{2} s_{3}+2 a_{3} s_{8}\right)}{12} & 0 & \frac{\lambda^{2}\left(s_{7}-1\right)}{2} & \frac{\lambda\left(s_{3}-1\right)}{4} & \frac{\lambda\left(s_{4}-1\right)}{4} & 0 & 0 & \frac{s_{7}-1}{2} & \frac{s_{8}-1}{6 \lambda} \\
\frac{\lambda^{3}\left(4-a_{2} s_{3}-2 a_{3} s_{8}\right)}{36} & 0 & -\frac{\lambda^{2}\left(s_{7}+1\right)}{6} & \frac{\lambda\left(s_{3}-1\right)}{36} & \frac{\lambda\left(s_{4}-1\right)}{4} & 0 & 0 & \frac{s_{7}-1}{6} & \frac{s_{8}-1}{18 \lambda} \\
0 & \frac{\lambda^{2}\left(2-s_{7}\right)}{6} & 0 & 0 & 0 & \frac{\lambda\left(s_{4}-1\right)}{2} & \frac{s_{7}-1}{6} & 0 & 0 \\
0 & \frac{\lambda^{3}\left(s_{7}-2\right)}{6} & 0 & 0 & 0 & \frac{\lambda^{2}\left(s_{4}-1\right)}{2} & \frac{\lambda\left(s_{7}-1\right)}{6} & 0 & 0 \\
\frac{\lambda^{4}\left(a_{2} s_{3}+a_{3} s_{8}\right)}{6} & 0 & \frac{\lambda^{3} s_{7}}{2} & \frac{\lambda^{2}\left(s_{4}-1\right)}{6} & \frac{\lambda^{2}\left(s_{4}-1\right)}{2} & 0 & 0 & \frac{\lambda\left(s_{7}-1\right)}{2} & \frac{s_{8}-1}{6} \\
-\frac{\lambda^{5}\left(a_{2} s_{3}+a_{3} s_{8}\right)}{6} & 0 & -\frac{\lambda^{4} s_{7}}{2} & \frac{\lambda^{3}\left(s_{4}-1\right)}{6} & \frac{\lambda^{3}\left(s_{4}-1\right)}{2} & 0 & 0 & \frac{\lambda^{2}\left(s_{7}-1\right)}{2} & \frac{s_{8}-1}{6 \lambda}
\end{array}\right) .
\end{aligned}
$$

- The right hand side $g^{0} \equiv M \xi^{1}-\partial_{t} m^{0}-B^{\alpha} \partial_{\alpha} m^{0}$ of the the relation (14) is admissible, i.e. the equation (14) admits at least one solution, if and only if the condition (34) is satisfied. With the previous data, this condition takes the form

$$
\partial_{t} \rho+\partial_{x} J_{x}+\partial_{y} J_{y}-\frac{1}{\lambda}\left[\partial_{t} J_{y}+\left(\frac{2}{3}+\frac{a_{2}}{6}\right) \lambda^{2} \partial_{y} \rho\right]=\mathrm{O}(\Delta t) .
$$

The above condition is a linear combination of the equivalent partial differential equations of the scheme at order 1 (see $e$. g. [18]). They are naturally satisfied. We observe that if the fields $J_{x}$ and $J_{y}$ represent given Dirichlet values of the boundary condition, the partial derivatives $\partial_{t} J_{x}, \partial_{t} J_{y}, \partial_{x} J_{x}$ and $\partial_{x} J_{y}$ are defined without ambiguity by the problem. Nevertheless, the partial derivatives $\partial_{y} J_{x}$ and $\partial_{y} J_{y}$ are Neumann boundary conditions that are not a part of the given boundary conditions. In this contribution, we consider 
these data to be the exact values of the underlying partial differential equation. The solution $m^{1}$ of the equation (14) is given by

$$
m^{1}=\left(\begin{array}{c}
0 \\
-\left(2 \sigma_{7}+\frac{3}{2}\right) \partial_{t} J_{x}+\frac{\lambda}{2} \partial_{y} J_{x}+\frac{\lambda^{2}}{12}\left[\left(2 a_{2}+4 a_{3}-8\right) \sigma_{7}-3 a_{2}-12\right] \partial_{x} \rho \\
\frac{\lambda}{2} \partial_{y} J_{y}-\frac{1}{2} \partial_{t} J_{y}-\frac{\lambda^{2}}{12}\left(4+a_{2}\right) \partial_{y} \rho \\
\frac{\lambda^{2}\left(2 \sigma_{3}+1\right)}{6\left(2 \sigma_{7}+1\right)}\left[\left(3 a_{2}+2 a_{3}+4\right) \sigma_{7} \lambda \partial_{y} \rho-a_{2}\left(6 \sigma_{7}+3\right) \partial_{t} \rho\right] \\
\frac{\lambda^{2}}{3}\left(2 \sigma_{4}+1\right)\left(-\partial_{x} J_{x}+\partial_{y} J_{y}-\frac{1}{\lambda} \partial_{t} J_{y}\right)-\frac{\lambda^{3}\left(2 \sigma_{4}+1\right)}{18\left(2 \sigma_{7}+1\right)}\left[\sigma_{7}\left(-a_{2}-2 a_{3}+4\right)+a_{2}+4\right] \partial_{y} \rho \\
\frac{\lambda^{2}}{6}\left(2 \sigma_{4}+1\right)\left(-\partial_{y} J_{x}-\partial_{x} J_{y}+\frac{3}{\lambda} \partial_{t} J_{x}\right)+\frac{\lambda^{3}\left(2 \sigma_{4}+1\right)}{12}\left(4+a_{2}\right) \partial_{x} \rho \\
\frac{\lambda^{2}}{2}\left(-\lambda \partial_{y} J_{x}+\left(8 \sigma_{7}+5\right) \partial_{t} J_{x}\right)-\frac{\lambda^{4}}{6}\left[\left(2 a_{2}+4 a_{3}-8\right) \sigma_{7}-a_{2}+a_{3}-8\right] \partial_{x} \rho \\
\frac{\lambda^{2}}{2}\left(\partial_{t} J_{y}-\lambda \partial_{y} J_{y}\right)-\frac{\lambda^{4}}{6}\left(a_{2}+a_{3}\right) \partial_{y} \rho \\
\left.\frac{\lambda^{4}}{12} \frac{2 \sigma_{8}+1}{2 \sigma_{7}+1}\left[4 \lambda \sigma_{7}\left(a_{2}+a_{3}\right)-a_{2}-4\right) \partial_{y} \rho-6 a_{3}\left(2 \sigma_{7}+1\right) \partial_{t} \rho\right]+\frac{\lambda^{3}\left(2 \sigma_{8}+1\right)}{2}\left(-\partial_{t} J_{y}+\partial_{y} J_{y} \lambda+\lambda \partial_{x} J_{x}\right)
\end{array}\right)
$$

The matrices $\widetilde{B}^{\alpha, \beta}$ introduced in (15) take the following expressions for the previous bounce-back problem with the D2Q9 scheme:

$\widetilde{B}^{1,1}=\left(\begin{array}{ccccccccc}\frac{\lambda^{2}\left(8+a_{2} s_{3}-a_{3} s_{8}\right)}{18} & 0 & \frac{\lambda\left(s_{7}-2\right)}{6} & \frac{(1-s 3)}{18} & \frac{(1-s 4)}{2} & 0 & 0 & \frac{(s 7-1)}{6 \lambda} & \frac{(s 8-1)}{18 \lambda^{2}} \\ 0 & \frac{\lambda^{2}\left(4+s_{7}\right)}{6} & 0 & 0 & 0 & \frac{\lambda\left(s_{4}-1\right)}{2} & \frac{\left(s_{7}-1\right)}{6} & 0 & 0 \\ -\frac{\lambda^{3}\left(4+2 a_{2} s_{3}+a_{3} s_{8}\right)}{18} & 0 & \frac{\lambda^{2}\left(2-s_{7}\right)}{6} & \frac{\lambda\left(s_{3}-1\right)}{9} & 0 & 0 & 0 & \frac{(1-s 7)}{6} & \frac{(s 8-1)}{18 \lambda} \\ \frac{\lambda^{4}\left(4+5 a_{2} s_{3}+4 a_{3} s_{8}\right)}{18} & 0 & \frac{\lambda^{3}\left(s_{7}-2\right)}{3} & \frac{5 \lambda^{2}\left(1-s_{3}\right)}{18} & \frac{\lambda^{2}(s 4-1)}{2} & 0 & 0 & \frac{\lambda(s 7-1)}{3} & \frac{2\left(1-s_{8}\right)}{9} \\ \frac{\lambda^{4}\left(4-a_{2} s_{3}-2 a_{3} s_{8}\right)}{18} & 0 & 0 & \frac{\lambda^{2}\left(s_{3}-1\right)}{18} & \frac{\lambda^{2}\left(1-s_{4}\right)}{2} & 0 & 0 & 0 & \frac{\left(s_{8}-1\right)}{9} \\ 0 & \frac{\lambda^{3}\left(s_{7}-2\right)}{6} & 0 & 0 & 0 & \frac{\lambda^{2}\left(1-s_{4}\right)}{2} & \frac{\lambda\left(s_{7}-1\right)}{6} & 0 & 0 \\ 0 & -\frac{\lambda^{4}\left(2+5 s_{7}\right)}{6} & 0 & 0 & 0 & \frac{\lambda^{3}\left(s_{4}-1\right)}{2} \frac{5 \lambda^{2}\left(1-s_{7}\right)}{6} & 0 & 0 \\ \frac{-\frac{\lambda^{5}\left(4+2 a_{2} s_{3}+a_{3} s_{8}\right)}{18}}{0} & \frac{\lambda^{4}\left(2-s_{7}\right)}{6} & \frac{\lambda^{3}\left(s_{3}-1\right)}{9} & 0 & 0 & 0 & \frac{\lambda^{2}(1-s 7)}{6} & \frac{\lambda(s 8-1)}{18} \\ \frac{\lambda^{6}\left(-4+4 a_{2} s_{3}+5 a_{3} s_{8}\right)}{18} & 0 & \frac{\lambda^{5}\left(s_{7}-2\right)}{6} & \frac{2 \lambda^{4}\left(1-s_{3}\right)}{9} & \lambda^{4}(s 4-1) & 0 & 0 & \frac{\lambda^{3}(s 7-1)}{6} & \frac{5 \lambda^{2}\left(1-s_{8}\right)}{18}\end{array}\right)$

$\widetilde{B}^{2,2}=\left(\begin{array}{ccccccccc}\frac{\lambda^{2}}{12}\left(4+a_{2} s_{3}\right) & 0 & -\frac{1}{2 \lambda} & \frac{\left(1-s_{3}\right)}{12} & \frac{s_{4}-1}{4} & 0 & 0 & 0 & 0 \\ 0 & \frac{\lambda^{2}\left(2-s_{7}\right)}{6} & 0 & 0 & 0 & \frac{\lambda\left(s_{4}-1\right)}{2} & \frac{1-s_{7}}{6} & 0 & 0 \\ -\frac{\lambda^{3}\left(4+a_{2} s_{3}\right)}{12} & 0 & \frac{\lambda^{2}}{2} & \frac{\lambda\left(s_{3}-1\right)}{12} & \frac{\lambda\left(1-s_{4}\right)}{4} & 0 & 0 & 0 & 0 \\ \frac{\lambda^{4}\left(4+3 a_{2}+2 a_{3} s_{8}\right)}{12} & 0 & \frac{\lambda^{3}\left(s_{7}-1\right.}{2} & \frac{\lambda^{2}\left(1-s_{3}\right)}{4} & \frac{\lambda^{2}\left(1-s_{4}\right)}{4} & 0 & 0 & \frac{\lambda\left(s_{7}-1\right)}{2} & \frac{\left(1-s_{8}\right)}{6} \\ \frac{\lambda^{4}\left(-4+a_{2} s_{3}+2 a_{3} s_{8}\right)}{36} & 0 & \frac{\lambda^{3}\left(1+s_{7}\right)}{6} & \frac{\lambda^{2}\left(1-s_{3}\right)}{36} & \frac{\lambda^{2}\left(1-s_{4}\right)}{4} & 0 & 0 & \frac{\lambda\left(s_{7}-1\right)}{6} & \frac{\left(1-s_{8}\right)}{18} \\ 0 & \frac{\lambda^{3}\left(s_{7}-2\right)}{6} & 0 & 0 & 0 & \frac{\lambda^{2}\left(1-s_{4}\right)}{2} & \frac{\lambda\left(s_{7}-1\right)}{6} & 0 & 0 \\ 0 & \frac{\lambda^{4}\left(2-s_{7}\right)}{6} & 0 & 0 & 0 & \frac{\lambda^{3}\left(s_{4}-1\right)}{2} & \frac{\lambda^{2}\left(1-s_{7}\right)}{6} & 0 & 0 \\ \frac{\lambda^{5}\left(a_{2} s_{3}+a_{3} s_{8}\right)}{6} & 0 & -\frac{\lambda^{4} s_{7}}{2} & \frac{\lambda^{3}\left(s_{3}-1\right)}{6} & \frac{\lambda^{3}\left(s_{4}-1\right)}{2} & 0 & 0 & \frac{\lambda^{2}\left(1-s_{7}\right)}{2} & \frac{\lambda\left(s_{8}-1\right)}{6} \\ \frac{\lambda^{5}\left(a_{2} s_{3}+a_{3} s_{8}\right)}{6} & 0 & \frac{\lambda^{4} s_{7}}{2} & \frac{\lambda^{3}\left(1-s_{3}\right)}{6} & \frac{\lambda^{3}\left(1-s_{4}\right)}{2} & 0 & 0 & \frac{\lambda^{2}\left(s_{7}-1\right)}{2} & \frac{\lambda\left(1-s_{8}\right)}{6}\end{array}\right)$ 
$\widetilde{B}^{1,2}=\left(\begin{array}{c}0 \\ -\frac{\lambda^{3}\left(4+2 a_{2} s_{3}+a_{3} s_{8}\right)}{18} \\ 0 \\ 0 \\ 0 \\ \frac{\lambda^{4}\left(4+2 a_{2} s_{3}+a_{3} s_{8}\right)}{18} \\ -\frac{\lambda^{5}\left(4+2 a_{2} s_{3}+a_{3} s_{8}\right)}{18} \\ 0 \\ 0\end{array}\right.$

$\begin{array}{cc}\frac{\lambda\left(s_{7}-2\right)}{6} & 0 \\ 0 & \frac{\lambda^{2}\left(2-s_{7}\right)}{6} \\ \frac{\lambda^{2}\left(2-s_{7}\right)}{6} & 0 \\ \frac{\lambda^{3}\left(s_{7}-2\right)}{3} & 0 \\ 0 & 0 \\ 0 & \frac{\lambda^{3}\left(s_{7}-2\right)}{6} \\ 0 & \frac{\lambda^{4}\left(2-s_{7}\right)}{6} \\ \frac{\lambda^{4}\left(2-s_{7}\right)}{6} & 0 \\ \frac{\lambda^{5}\left(s_{7}-2\right)}{6} & 0\end{array}$

$\begin{array}{ccc}0 & 0 & \frac{1-s_{4}}{2} \\ \frac{\lambda\left(s_{3}-1\right)}{9} & 0 & 0 \\ 0 & 0 & \frac{\lambda\left(s_{4}-1\right)}{2}, \frac{\left(1-s_{7}\right)}{6} \\ 0 & 0 & \lambda^{2}(1-s 4) \\ 0 & 0 & 0 \\ \frac{\lambda^{2}\left(1-s_{3}\right)}{9} & 0 & 0 \\ \frac{\lambda^{3}\left(s_{3}-1\right)}{9} & 0 & 0 \\ 0 & 0 & \frac{\lambda^{3}\left(s_{4}-1\right)}{2} \\ 0 & 0 & \frac{\lambda^{4}\left(1-s_{4}\right)}{2}\end{array}$

$\frac{\lambda\left(s_{7}-1\right)}{6}$
0
0
$\frac{\lambda\left(s_{7}-1\right)}{3}$
0
0
0
$\frac{\lambda^{2}\left(1-s_{7}\right)}{6}$
$\frac{\lambda^{3}\left(s_{7}-1\right)}{6}$

0
$\frac{\left(1-s_{7}\right)}{6}$
0
0
0
$\frac{\lambda\left(s_{7}-1\right)}{6}$
$\frac{\lambda^{2}\left(1-s_{7}\right)}{6}$
0
0

$$
\left.\begin{array}{c}
0 \\
\frac{\left(s_{8}-1\right)}{\lambda 18} \\
0 \\
0 \\
\frac{1-s_{8}}{18} \\
\frac{\lambda\left(s_{8}-1\right)}{18} \\
0 \\
0
\end{array}\right)
$$

- The condition $(34)$ at order 2 on $(\Delta t)$ takes the form:

$$
\left\{\begin{aligned}
\partial_{t} \rho & +\partial_{x} J_{x}+\partial_{y} J_{y}-\frac{1}{\lambda}\left[\partial_{t} J_{y}+\left(\frac{2}{3}+\frac{a_{2}}{6}\right) \lambda^{2} \partial_{y} \rho\right]+\frac{\Delta t}{\lambda}\left[\alpha_{x y} \partial_{x} \partial_{y} J_{x}\right. \\
& \left.+\beta_{t t} \partial_{t}^{2} J_{y}+\beta_{t y} \partial_{t} \partial_{y} J_{y}+\beta_{x x} \partial_{x}^{2} J_{y}+\beta_{y y} \partial_{y}^{2} J_{y}+\gamma_{t t} \partial_{t}^{2} \rho+\gamma_{x x} \partial_{x}^{2} \rho+\gamma_{y y} \partial_{y}^{2} \rho\right]=\mathrm{O}\left(\Delta t^{2}\right)
\end{aligned}\right.
$$

where

$$
\left\{\begin{array}{l}
\alpha_{x y}=-\frac{\lambda}{12}\left(2 a_{2} \sigma_{3}-a_{2}-10\right), \quad \beta_{t t}=-\frac{1}{2 \lambda}, \quad \beta_{t y}=-\frac{1}{2}, \quad \beta_{x x}=\frac{\lambda}{3} \sigma_{4} \\
\beta_{x y}=-\frac{\lambda}{12}\left(2 a_{2} \sigma_{3}-4 \sigma_{4}-a_{2}-10\right), \quad \gamma_{t t}=\frac{1}{2}, \quad \gamma_{x x}=\frac{\lambda^{2}}{12}\left(6 a_{2} \sigma_{7}+4 a_{3} \sigma_{7}+8 \sigma_{7}-a_{2}-4\right) \\
\gamma_{y y}=-\frac{\lambda^{2}}{36\left(2 \sigma_{7}+1\right)}\left[\left(6 a_{2}+4 a_{3}+8\right) \sigma_{3} \sigma_{7}-\left(6 a_{2}+4 a_{3}+8\right) \sigma_{4} \sigma_{7}+\left(6 a_{2}+24\right) \sigma_{7}+3 a_{2}+12\right]
\end{array}\right.
$$

We observe that the left hand side of the equation (38) is exactly a linear combination of the equivalent equations of the internal scheme at second order for mass and momentum, [18].

\section{REFERENCES}

[1] M. Bouzidi, M. Firdaous, P. Lallemand, "Momentum transfer of a Boltzmann-lattice fluid with boundaries", Physics of Fluids, vol. 13, p. 3452-3459, 2001.

[2] A. Caiazzoa, M. Junk, Boundary forces in lattice Boltzmann: Analysis of Momentum Exchange algorithm, Computers \& Mathematics with Applications, 55, p. 1415-1423, 2008.

[3] Y. Chen, Q. Cai, Z. Xia, M. Wang, S. Chen, Momentum-exchange method in lattice Boltzmann simulations of particle-fluid interactions, Phys. Rev. E, 88, 013303, 2013.

[4] F. Dubois, Equivalent partial differential equations of a lattice Boltzmann scheme, Computers 83 Mathematics with Applications, 55, p. 1441-1449, 2008.

[5] F. Dubois, P. Lallemand, "Towards higher order lattice Boltzmann schemes", Journal of Statistical Mechanics: Theory and Experiment, P06006 doi: 10.1088/1742-5468/2009/06/P06006, 2009.

[6] F. Dubois, P. Lallemand, M. M. Tekitek, "Using the lattice Boltzmann scheme for anisotropic diffusion problems", Finite Volumes for complex Applications V, R. Eymard, J.M. Hérard (Eds.), Wiley, p. 351-358, 2008.

[7] F. Dubois, P. Lallemand, M. M. Tekitek, "On a superconvergent lattice Boltzmann boundary scheme", Computers and Mathematics with Applications, vol. 59, p. 2141-2149, 2010.

[8] F. Dubois, P. Lallemand, M.M. Tekitek, "Taylor Expansion Method for Linear Lattice Boltzmann Schemes with an External Force: Application to Boundary Conditions", Lecture Notes in Computational Science and Engineering, vol. 99, p. 89-107. Edited by R. Abgrall et al., Springer, 2014.

[9] I. Ginzburg, P. Adler, "Boundary flow condition analysis for the three-dimensional lattice Boltzmann model", Journal of Physics II France, vol. 4, p. 191-214, 1994.

[10] X. He, G. Doolen, "Lattice Boltzmann method on curvilinear coordinates system: flow around a circular cylinder", Journal of Computational Physics, vol. 134, p. 306-315, 1997.

[11] X. He, Q. Zou, L.-S. Luo, M. Dembo, "Analytic solutions and analysis on non-slip boundary condition for the lattice Boltzmann BGK model", Journal of Statistical Physics, 87, p. 115-136, 1997. 
[12] D. d'Humières, "Generalized Lattice-Boltzmann Equations", in Rarefied Gas Dynamics: Theory and Simulations, vol. 159 of AIA A Progress in Aeronautics and Astronautics, p. 450-458, 1992.

[13] D. d'Humières, I. Ginzburg, "Multi-reflection boundary conditions for lattice Boltzmann models", Physical Review E, vol. 68, issue 6, p. 066614 (30 pages), 2003.

[14] T. Inamuro, K. Maeba, F. Ogino, "Flow between parallel walls containing the lines of neutrally buoyant circular cylinders", International Journal of Multiphase Flow, vol. 26, p. 1981-2004, 2000.

[15] M. Junk, A. Klar, "Discretizations for the incompressible Navier-Stokes equations based on the lattice Boltzmann method", SIAM Journal on Scientific Computing, vol. 22, p. 1-19, 2000.

[16] A. J. C. Ladd, "Numerical Simulations of Particulate Suspensions via a discretized Boltzmann Equation. Part I. Theoretical Foundation", Journal of Fluid Mechanics, vol. 271, p. 285-309, 1994.

[17] A. J. C. Ladd, "Numerical Simulations of Particulate Suspensions via a discretized Boltzmann Equation. Part II. Numerical results", Journal of Fluid Mechanics, vol. 271, p. 311-339, 1994.

[18] P. Lallemand, L. Luo, "Theory of the lattice Boltzmann method: Dispersion, dissipation, isotropy, Galilean invariance, and stability", Physical Review E, vol. 61, p. 6546-6562, 2000.

[19] H. Li, X. Lu, H. Fang, Y. Qian, "Force evaluations in lattice Boltzmann simulations with moving boundaries in two dimension", Phys. Rev. E, 70, 026701, 2004

[20] R. Mei, D. Yu, W. Shyy, L. Luo, "Force evaluation in the lattice Boltzmann method involving curved geometry", Physical Review E, vol. 65, 041203, 2002.

[21] Q. Zou, X. He, "On pressure and velocity boundary conditions for the lattice Boltzmann BGK model", Physics of Fluids, vol. 9, p. 1591-1598, 1997. 